\title{
Sawyer-type inequalities for Lorentz spaces
}

\author{
Carlos Pérez ${ }^{1} \cdot$ Eduard Roure-Perdices $^{2}$
}

Received: 6 April 2020 / Revised: 9 July 2021 / Accepted: 12 July 2021 /

Published online: 21 July 2021

(C) The Author(s) 2021

\section{Abstract}

The Hardy-Littlewood maximal operator $M$ satisfies the classical Sawyer-type estimate

$$
\left\|\frac{M f}{v}\right\|_{L^{1, \infty}(u v)} \leq C_{u, v}\|f\|_{L^{1}(u)},
$$

where $u \in A_{1}$ and $u v \in A_{\infty}$. We prove a novel extension of this result to the general restricted weak type case. That is, for $p>1, u \in A_{p}^{\mathcal{R}}$, and $u v^{p} \in A_{\infty}$,

$$
\left\|\frac{M f}{v}\right\|_{L^{p, \infty}\left(u v^{p}\right)} \leq C_{u, v}\|f\|_{L^{p, 1}(u)} .
$$

From these estimates, we deduce new weighted restricted weak type bounds and Sawyer-type inequalities for the $m$-fold product of Hardy-Littlewood maximal operators. We also present an innovative technique that allows us to transfer such estimates to a large class of multi-variable operators, including $m$-linear CalderónZygmund operators, avoiding the $A_{\infty}$ extrapolation theorem and producing many estimates that have not appeared in the literature before. In particular, we obtain a new characterization of $A_{p}^{\mathcal{R}}$. Furthermore, we introduce the class of weights that characterizes the restricted weak type bounds for the multi(sub)linear maximal operator $\mathcal{M}$, denoted by $A_{\mathbf{P}}^{\mathcal{R}}$, establish analogous bounds for sparse operators and $m$-linear Calderón-Zygmund operators, and study the corresponding multi-variable

Communicated by Loukas Grafakos.

Eduard Roure-Perdices

eduardroure@protonmail.ch

Carlos Pérez

cperez@bcamath.org

1 Department of Mathematics, University of the Basque Country, Ikerbasque and BCAM, Bilbao, Spain

2 Departament de Matemàtiques i Informàtica, Universitat de Barcelona, 08007 Barcelona, Spain 
Sawyer-type inequalities for such operators and weights. Our results combine mixed restricted weak type norm inequalities, $A_{p}^{\mathcal{R}}$ and $A_{\mathbf{P}}^{\mathcal{R}}$ weights, and Lorentz spaces.

Mathematics Subject Classification 42B25 - 46E30

\section{Introduction}

"Sawyer-type inequalities" is a terminology coined in the paper [19], where the authors prove that if $u \in A_{1}$, and $v \in A_{1}$ or $u v \in A_{\infty}$, then

$$
u v\left(\left\{x \in \mathbb{R}^{n}: \frac{|T(f v)(x)|}{v(x)}>t\right\}\right) \leq \frac{C}{t} \int_{\mathbb{R}^{n}}|f(x)| u(x) v(x) d x, \quad t>0,
$$

where $T$ is either the Hardy-Littlewood maximal operator or a linear CalderónZygmund operator. This result extends some questions previously considered by B. Muckenhoupt and R. Wheeden in [48], and solves in the affirmative a conjecture formulated by E. Sawyer in [54] concerning the Hilbert transform. These problems were advertised by B. Muckenhoupt in [47], where the terminology "mixed type norm inequalities" was introduced and was also used since then in other papers like [2] or [44]. In general, this terminology refers to certain weighted estimates for some classical operators $T$, where a weight $v$ is included in their level sets; that is,

$$
\left\{x \in \mathbb{R}^{n}: \frac{|T f(x)|}{v(x)}>t\right\}, \quad t>0 .
$$

The structure of such sets makes it impossible, or very difficult, to use classical tools to measure them, such as Vitali's covering lemma or interpolation theorems.

In this paper, we consider mixed restricted weak type norm inequalities, or Sawyertype inequalities for Lorentz spaces; that is, we study estimates of the form

$$
w\left(\left\{x \in \mathbb{R}^{n}: \frac{|T f(x)|}{v(x)}>t\right\}\right)^{1 / p} \leq \frac{C}{t}\|f\|_{L^{p, 1}(u)}, \quad t>0
$$

where $p \geq 1, T$ is a classical operator, and $u, v, w$ are weights. We also consider extensions of such inequalities to the multi-variable setting. Our goal is to prove estimates like (1.3) for sub-linear and multi-sub-linear maximal operators, and multilinear Calderón-Zygmund operators. Observe that in the classical situation, namely when $u=w, v \approx 1$, and $T$ is either the Hardy-Littlewood maximal operator or a linear Calderón-Zygmund operator, the inequality (1.3) holds if $w \in A_{p}^{\mathcal{R}}$ (some authors use the notation $A_{p, 1}$ for this class of weights, as in [16]). The case when $v \not \approx 1$ is much more difficult, and in what follows, we will study it in great detail.

The starting point of this paper and our primary motivation to consider Sawyertype inequalities for Lorentz spaces comes from the study of the $m$-fold product of 
Hardy-Littlewood maximal operators,

$$
M^{\otimes}(\mathbf{f})(x):=\prod_{i=1}^{m} M f_{i}(x), \quad x \in \mathbb{R}^{n} .
$$

M. J. Carro and E. R. P. proved in [14] that for $1 \leq p_{1}, \ldots, p_{m}<\infty$ and $\frac{1}{p}=$ $\frac{1}{p_{1}}+\cdots+\frac{1}{p_{m}}$, and weights $w_{1}, \ldots, w_{m} \in A_{\infty}$ and $v_{\mathbf{w}}:=w_{1}^{p / p_{1}} \ldots w_{m}^{p / p_{m}}$, a necessary condition to have

$$
M^{\otimes}: L^{p_{1}, 1}\left(w_{1}\right) \times \cdots \times L^{p_{m}, 1}\left(w_{m}\right) \longrightarrow L^{p, \infty}\left(v_{\mathbf{w}}\right)
$$

is that $w_{i} \in A_{p_{i}}^{\mathcal{R}}$, for $i=1, \ldots, m$. They left as an open question to prove that this last condition is also sufficient for (1.4) to hold. It is reasonable to think that this may indeed be true since the endpoint case was proved in [36]. That is, for weights $w_{1}, \ldots, w_{m} \in A_{1}$, we have that

$$
M^{\otimes}: L^{1}\left(w_{1}\right) \times \cdots \times L^{1}\left(w_{m}\right) \longrightarrow L^{1 / m, \infty}\left(w_{1}^{1 / m} \ldots w_{m}^{1 / m}\right) .
$$

To prove this result, one has to control the following quantity for $t>0$, which is related to the level sets (1.2):

$$
v_{\mathbf{w}}\left(\left\{M^{\otimes}(\mathbf{f})>t\right\}\right)=v_{\mathbf{w}}\left(\left\{x \in \mathbb{R}^{n}: M f_{i}(x)>\frac{t}{\prod_{j \neq i} M f_{j}(x)}\right\}\right),
$$

where $v_{\mathbf{w}}=w_{1}^{1 / m} \ldots w_{m}^{1 / m}$. This is achieved by applying the classical Sawyer-type inequality (1.1) for the Hardy-Littlewood maximal operator $M$ in combination with the observation that for locally integrable functions $h_{1}, \ldots, h_{k}, \prod_{j=1}^{k}\left(M h_{j}\right)^{-1} \in R H_{\infty}$, with constant depending only on $k$ and the dimension $n$.

As we will show in Theorem 3, it turns out that the bound (1.4) holds if $w_{i} \in A_{p_{i}}^{\mathcal{R}}$, for $i=1, \ldots, m$, solving in the affirmative the open question in [14] and completing the characterization of the restricted weak type bounds of $M^{\otimes}$ for $A_{\infty}$ weights. The strategy that we follow is similar to the one in [36] for the endpoint case (1.5), but we have to replace the classical Sawyer-type inequality (1.1) by the estimate obtained in Theorem 2, which is a new restricted weak Sawyer-type inequality involving the class of weights $A_{p}^{\mathcal{R}}$. That is,

$$
\left\|\frac{M f}{v}\right\|_{L^{p, \infty}\left(u v^{p}\right)} \leq C_{u, v}\|f\|_{L^{p, 1}(u)},
$$

for $p>1, u \in A_{p}^{\mathcal{R}}$, and $u v^{p} \in A_{\infty}$. The $A_{p}^{\mathcal{R}}$ condition on the weight $u$ is a natural assumption since it is necessary when $v \approx 1$. In Lemma 3 we also manage to track the dependence of the constant $C_{u, v}$ on the weights $u$ and $u v^{p}$, even in the endpoint case $p=1$, refining the bound (1.1) in [19]. 
There is no reason to restrict ourselves to the study of one-variable Sawyer-type inequalities. Quite recently, the bound (1.1) has been extended to the multi-variable setting in [40]. More precisely, for weights $w_{1}, \ldots, w_{m} \in A_{1}$, and $v \in A_{\infty}$,

$$
\left\|\frac{\mathcal{M}(\mathbf{f})}{v}\right\|_{L^{1 / m, \infty}\left(v_{\mathbf{w}} v^{1 / m}\right)} \leq\left\|\frac{\prod_{i=1}^{m} M f_{i}}{v}\right\|_{L^{1 / m, \infty}\left(v_{\mathbf{w}} v^{1 / m}\right)} \lesssim \prod_{i=1}^{m}\left\|f_{i}\right\|_{L^{1}\left(w_{i}\right)} .
$$

Inspired by this result, we follow a similar approach to extend our Sawyer-type inequality (1.6) to the multi-variable setting, obtaining a generalization of (1.7) in Theorem 4. That is, for weights $w_{1}, \ldots, w_{m}$ and $v$ such that for $i=1, \ldots, m, w_{i} \in A_{p_{i}}^{\mathcal{R}}$ and $w_{i} v^{p_{i}} \in A_{\infty}$,

$$
\left\|\frac{\mathcal{M}(\mathbf{f})}{v}\right\|_{L^{p, \infty}\left(v_{\mathbf{w}} v^{p}\right)} \leq\left\|\frac{\prod_{i=1}^{m} M f_{i}}{v}\right\|_{L^{p, \infty}\left(v_{\mathbf{w}} v^{p}\right)} \lesssim \prod_{i=1}^{m}\left\|f_{i}\right\|_{L^{p_{i}, 1}\left(w_{i}\right)} .
$$

Observe that this result is an extension of (1.4). To our knowledge, this multi-variable mixed restricted weak type inequalities for maximal operators involving the $A_{p}^{\mathcal{R}}$ condition on the weights have not been previously studied, and we found no record of them being conjectured in the literature.

Motivated by the conjecture of E. Sawyer in [54], we can ask ourselves if it is possible to obtain bounds like (1.8) for multi-linear Calderón-Zygmund operators $T$. Once again, the endpoint case $p_{1}=\cdots=p_{m}=1$ has already been considered and extensively investigated in [40]. There, it was shown that for weights $w_{1}, \ldots, w_{m} \in$ $A_{1}$, and $v_{\mathbf{w}} v^{1 / m} \in A_{\infty}$

$$
\left\|\frac{T(\mathbf{f})}{v}\right\|_{L^{1 / m, \infty}\left(v_{\mathbf{w}} v^{1 / m}\right)} \lesssim \prod_{i=1}^{m}\left\|f_{i}\right\|_{L^{1}\left(w_{i}\right)},
$$

as a corollary of (1.7), combined with a result in [49], that allows replacing $\mathcal{M}$ by $T$ using an extrapolation type argument based on the $A_{\infty}$ extrapolation theorem obtained in $[18,22]$. We succeed in our goal and manage to get an extension of (1.9) to the general restricted weak setting. In Theorem 6 we prove, among other things, that for weights $w_{1}, \ldots, w_{m}$ and $v$ such that for $i=1, \ldots, m, w_{i} \in A_{p_{i}}^{\mathcal{R}}$ and $w_{i} v^{p_{i}} \in A_{\infty}$, and some other technical hypotheses on the weights,

$$
\left\|\frac{T(\mathbf{f})}{v}\right\|_{L^{p, \infty}\left(v_{\mathbf{w}} v^{p}\right)} \lesssim \prod_{i=1}^{m}\left\|f_{i}\right\|_{L^{p_{i}, 1}\left(w_{i}\right)}
$$

To achieve this, we build upon (1.8), but unlike in [40], we manage to avoid the use of extrapolation arguments like the ones in [49]. Instead, we present in Theorem 5 a novel technique that allows us to replace $\mathcal{M}$ by $T$ exploiting the fine structure of the Lorentz space $L^{p, \infty}$, the $A_{p}^{\mathcal{R}}$ condition, and the recent advances in sparse domination.

One can even go further and consider inequalities like (1.10) assuming multivariable conditions on the weights involved, as it was done in [40] with the endpoint 
case $p_{1}=\cdots=p_{m}=1$ and weights in $A_{\mathbf{1}}$. In Sect. 5, we discuss our findings on this matter. There, we introduce the class of weights that characterizes the restricted weak type bounds of $\mathcal{M}$, denoted by $A_{\mathbf{P}}^{\mathcal{R}}$, study some of its properties, deduce the corresponding restricted weak type bounds for sparse operators and multilinear Calderón-Zygmund operators, and conjecture the main results on Sawyer-type inequalities with weights in $A_{\mathbf{P}}^{\mathcal{R}}$. It is worth mentioning that we couldn't find in the literature any trace of results like (1.10) involving $\mathcal{M}$ or multi-linear Calderón-Zygmund operators, $A_{p}^{\mathcal{R}}$ or $A_{\mathbf{P}}^{\mathcal{R}}$ weights, and mixed restricted weak type inequalities.

Curiously, we didn't find much about Sawyer-type inequalities for Lorentz spaces apart from the endpoint results studied in [2,19,24,39,40,44,47-49,54], and some endpoint estimates for multi-variable fractional operators (see [52]), multi-linear pseudo-differential operators (see [12]), and the Hardy averaging operator (see $[41,43])$. As we have seen before, these inequalities are fundamental to understand the behavior of the operator $M^{\otimes}$, but they appear naturally in the study of other classical operators, even in the one-variable case. Consider, for example, the case of the Hilbert transform $H$. Indeed, if $p>1$ and $w \in A_{p}^{\mathcal{R}}$, it is well-known that $H: L^{p, 1}(w) \longrightarrow L^{p, \infty}(w)$. Hence, duality, linearity, and self-adjointness of $H$ yield

$$
\left\|\frac{H(f w)}{w}\right\|_{L^{p^{\prime}, \infty}(w)} \leq C_{w}\|f\|_{L^{p^{\prime}, 1}(w)} .
$$

This is an example of an estimate like (1.3) involving the $A_{p}^{\mathcal{R}}$ condition on the weights and obtained almost without effort. The same inequality holds for the HardyLittlewood maximal operator $M$, but we cannot use the same argument, as shown in [15]. In Theorem 7 we will generalize such a result for $M$, obtaining as a particular case, a new characterization of $A_{p}^{\mathcal{R}}$ and an alternative proof of the result in [15]. In $[29,35]$, one can find similar endpoint estimates for Calderón-Zygmund operators, with $p^{\prime}=1$ and $w \in A_{1}$ (see also [11,50,51,55,56]).

Sawyer-type inequalities also arise in the broadly studied topic involving commutators of linear operators $T$ with a $B M O$ function $b$, although we will not deal with them in this paper. The crucial initial observation is that we can write $[b, T]$ as a complex integral operator using Cauchy's integral theorem, obtaining that for $\varepsilon>0$,

$$
[b, T] f=\frac{1}{2 \pi i} \int_{\{z \in \mathbb{C}:|z|=\varepsilon\}} \frac{T_{z}(f)}{z^{2}} d z,
$$

where

$$
T_{z}(f):=e^{z b} T\left(\frac{f}{e^{z b}}\right), \quad z \in \mathbb{C} .
$$

This approach was introduced in the celebrated paper [17] and was further developed in [1]. In the context of Lorentz spaces, for $p>1$ and a weight $w$, and in virtue of 
Minkowski's inequality, we get that for any $\varepsilon>0$,

$$
\|[b, T] f\|_{L^{p, \infty}(w)} \leq \frac{1}{\varepsilon} \sup _{z \in \mathbb{C}:|z|=\varepsilon}\left\|T_{z}(f)\right\|_{L^{p, \infty}(w)} .
$$

Since $b \in B M O$, as a consequence of the John-Nirenberg inequality, there is a constant $s_{0}>0$ such that for $|z| \leq s_{0}, v^{-1}:=\left|e^{z b}\right|=e^{\Re(z) b} \in A_{p}$, and hence, it is possible to deduce weighted inequalities for commutators from estimates of the form

$$
\left\|\frac{T(f v)}{v}\right\|_{L^{p, \infty}(w)} \lesssim\|f\|_{X}
$$

for a norm or a quasi-norm $\|\cdot\|_{X}$, and $v^{-1} \in A_{p}$. Further results for commutators involving Sawyer-type inequalities can be found in [6,7] (see also [8,9]).

Recently, E. R. P. has shown in [53] that Sawyer-type inequalities for Lorentz spaces play a fundamental role in the extension to the multi-variable setting of the restricted weak type Rubio de Francia's extrapolation presented in $[13,15]$. His approach suggests that Conjecture 1 will be crucial for proving multi-variable extrapolation theorems involving weights in $A_{\mathbf{P}}^{\mathcal{R}}$.

\section{Preliminaries}

\subsection{Lorentz spaces and classical weights}

Let us recall the definition of the Lebesgue and Lorentz spaces (see [4]). For $p>0$ and an arbitrary measure space $(X, v), L^{p, 1}(v)$ is the Lorentz space of $v$-measurable functions such that

$$
\|f\|_{L^{p, 1}(v)}:=p \int_{0}^{\infty} \lambda_{f}^{v}(y)^{1 / p} d y=\int_{0}^{\infty} f_{v}^{*}(t) t^{1 / p} \frac{d t}{t}<\infty,
$$

$L^{p}(v)$ is the Lebesgue space of $v$-measurable functions such that

$$
\|f\|_{L^{p}(v)}:=\left(\int_{X}|f|^{p} v\right)^{1 / p}<\infty \quad(\text { or } v-\underset{X}{\operatorname{ess} \sup }|f|<\infty \text { if } p=\infty)
$$

and $L^{p, \infty}(\nu)$ is the Lorentz space of $\nu$-measurable functions such that

$$
\|f\|_{L^{p, \infty}(v)}:=\sup _{y>0} y \lambda_{f}^{\nu}(y)^{1 / p}=\sup _{t>0} t^{1 / p} f_{v}^{*}(t)<\infty
$$

where $f_{v}^{*}$ is the decreasing rearrangement of $f$ with respect to $v$, defined by

$$
f_{v}^{*}(t):=\inf \left\{y>0: \lambda_{f}^{v}(y) \leq t\right\}, \quad \lambda_{f}^{v}(t):=v(\{x \in X:|f(x)|>t\}) .
$$


If $p \geq 1$, then $L^{p, 1}(v) \hookrightarrow L^{p}(v) \hookrightarrow L^{p, \infty}(v)$. Given a $\sigma$-finite measure space $(X, v)$, and parameters $0<r<p<\infty$, the quantity

$$
\|f\|_{L^{p, \infty}(v)}:=\sup _{0<\nu(E)<\infty} v(E)^{\frac{1}{p}-\frac{1}{r}}\left(\int_{E}|f|^{r} d v\right)^{1 / r}
$$

satisfies that

$$
\|f\|_{L^{p, \infty}(v)} \leq\|f\|_{L^{p, \infty}(v)} \leq\left(\frac{p}{p-r}\right)^{1 / r}\|f\|_{L^{p, \infty}(v)} .
$$

This result is classical (see [27, Exercise 1.1.12]), and throughout this paper, we will refer to these inequalities as Kolmogorov's inequalities.

Given $f \in L_{\text {loc }}^{1}\left(\mathbb{R}^{n}\right)$, the Hardy-Littlewood maximal operator $M$ is defined by

$$
M f(x):=\sup _{Q \ni x} \frac{1}{|Q|} \int_{Q}|f(y)| d y, \quad x \in \mathbb{R}^{n},
$$

where the supremum is taken over all cubes $Q \subseteq \mathbb{R}^{n}$ containing $x$. Muckenhoupt studied the boundedness of $M$ on Lebesgue spaces $L^{p}(w)$ (see [45]). Given a positive and locally integrable function $w$, called weight, and $1<p<\infty$,

$$
M: L^{p}(w) \longrightarrow L^{p}(w)
$$

if, and only if $w \in A_{p}$; that is, if

$$
[w]_{A_{p}}:=\sup _{Q}\left(f_{Q} w\right)\left(f_{Q} w^{1-p^{\prime}}\right)^{p-1}<\infty
$$

where we use the notation $f_{Q} w=\frac{1}{|Q|} \int_{Q} w(x) d x$. Moreover, if $1 \leq p<\infty$,

$$
M: L^{p}(w) \longrightarrow L^{p, \infty}(w)
$$

if, and only if $w \in A_{p}$, where a weight $w \in A_{1}$ if

$$
[w]_{A_{1}}:=\sup _{Q}\left(f_{Q} w\right)\left\|\chi \chi_{Q} w^{-1}\right\|_{L^{\infty}(w)}=\sup _{Q}\left(f_{Q} w\right)(\underset{x \in Q}{\operatorname{ess} \inf } w(x))^{-1}<\infty .
$$

Buckley proved in [10] that for $1 \leq p<\infty$,

$$
\|M\|_{L^{p}(w) \rightarrow L^{p, \infty}(w)} \lesssim[w]_{A_{p}}^{1 / p},
$$

and if $p>1$, then

$$
\|M\|_{L^{p}(w) \rightarrow L^{p}(w)} \lesssim[w]_{A_{p}}^{\frac{1}{p-1}}
$$


The restricted weak type bounds of $M$ were studied in [16,31]. For $1 \leq p<\infty$,

$$
M: L^{p, 1}(w) \longrightarrow L^{p, \infty}(w)
$$

if, and only if $w \in A_{p}^{\mathcal{R}}$, where a weight $w$ is in $A_{p}^{\mathcal{R}}$ (also denoted by $A_{p, 1}$ ) if

$$
[w]_{A_{p}^{\mathcal{R}}}:=\sup _{Q} w(Q)^{1 / p} \frac{\left\|\chi Q^{w^{-1}}\right\|_{L^{p^{\prime}, \infty}(w)}}{|Q|}<\infty
$$

or equivalently, if

$$
\|w\|_{A_{p}^{\mathcal{R}}}:=\sup _{Q} \sup _{E \subseteq Q} \frac{|E|}{|Q|}\left(\frac{w(Q)}{w(E)}\right)^{1 / p}<\infty .
$$

Given a measurable set $E$, we write $w(E)=\int_{E} w(x) d x$. If $w=1$, we simply write $|E|$. We have that $[w]_{A_{p}^{\mathcal{R}}} \leq\|w\|_{A_{p}^{\mathcal{R}}} \leq p[w]_{A_{p}^{\mathcal{R}}}$. Moreover,

$$
\|M\|_{L^{p, 1}(w) \rightarrow L^{p, \infty}(w)} \approx[w]_{A_{p}^{\mathcal{R}}} .
$$

As usual, we write $A \lesssim B$ if there exists a positive constant $C>0$, independent of $A$ and $B$, such that $A \leq C B$. If the implicit constant $C$ depends on some parameter $\alpha$, we may write $\lesssim_{\alpha}$ at our discretion. If $A \lesssim B$ and $B \lesssim A$, then we write $A \approx B$.

We now give the definitions of some other classes of weights that will appear later. For more information about them, see $[19,21,23,26]$. Define the class of weights

$$
A_{\infty}:=\bigcup_{p \geq 1} A_{p}=\bigcup_{p \geq 1} A_{p}^{\mathcal{R}}
$$

A weight $w \in A_{\infty}$ if, and only if

$$
[w]_{A_{\infty}}:=\sup _{Q} \frac{1}{w(Q)} \int_{Q} M\left(w \chi_{Q}\right)<\infty
$$

This quantity is usually referred to as the Fujii-Wilson $A_{\infty}$ constant (see [25]). More generally, given a weight $u$, and $p>1$, we say that $w \in A_{p}(u)$ if

$$
[w]_{A_{p}(u)}:=\sup _{Q}\left(\frac{1}{u(Q)} \int_{Q} w u\right)\left(\frac{1}{u(Q)} \int_{Q} w^{1-p^{\prime}} u\right)^{p-1}<\infty
$$

and $w \in A_{1}(u)$ if

$$
[w]_{A_{1}(u)}:=\sup _{Q}\left(\frac{1}{u(Q)} \int_{Q} w u\right)\left\|\chi \chi_{Q} w^{-1}\right\|_{L^{\infty}(w u)}
$$




$$
=\sup _{Q}\left(\frac{1}{u(Q)} \int_{Q} w u\right)(\underset{x \in Q}{\operatorname{ess} \inf } w(x))^{-1}<\infty
$$

and as before, we define

$$
A_{\infty}(u):=\bigcup_{p \geq 1} A_{p}(u)
$$

If $u$ is a doubling weight for cubes in $\mathbb{R}^{n}$, and $w \in A_{\infty}(u)$, then

$$
[w]_{A_{\infty}(u)}:=\sup _{Q} \frac{1}{w u(Q)} \int_{Q} M_{u}\left(w \chi_{Q}\right) u<\infty
$$

where

$$
M_{u} f(x):=\sup _{Q \ni x} \frac{1}{u(Q)} \int_{Q}|f(y)| u(y) d y
$$

is the weighted Hardy-Littlewood maximal operator. If $p>1$, then $M_{u}$ is bounded on $L^{p}(w u)$ if, and only if $w \in A_{p}(u)$, provided that $u$ is doubling. Given $s>1$, we say that a weight $w \in R H_{S}$ if

$$
[w]_{R H_{s}}:=\sup _{Q} \frac{|Q|}{w(Q)}\left(f_{Q} w^{s}\right)^{1 / s}<\infty
$$

and $w \in R H_{\infty}$ if

$$
[w]_{R H_{\infty}}:=\sup _{Q} \frac{|Q|}{w(Q)}\|\chi Q w\|_{L^{\infty}\left(\mathbb{R}^{n}\right)}=\sup _{Q} \frac{|Q|}{w(Q)} \operatorname{ess} \sup _{x \in Q} w(x)<\infty
$$

We have that

$$
A_{\infty}=\bigcup_{1<s \leq \infty} R H_{s}
$$

In [36], the following multi-variable extension of the Hardy-Littlewood maximal operator was introduced in connection with the theory of multi-linear CalderónZygmund operators:

$$
\mathcal{M}(\mathbf{f}):=\sup _{Q} \prod_{i=1}^{m}\left(f_{Q}\left|f_{i}\right|\right) \chi_{Q},
$$

for $\mathbf{f}=\left(f_{1}, \ldots, f_{m}\right)$, with $f_{i} \in L_{l o c}^{1}\left(\mathbb{R}^{n}\right), i=1, \ldots, m$. Commonly, this operator is referred to as the curly operator. For exponents $1 \leq p_{1}, \ldots, p_{m}<\infty, \mathbf{P}=$ 
$\left(p_{1}, \ldots, p_{m}\right), \frac{1}{p}=\frac{1}{p_{1}}+\cdots+\frac{1}{p_{m}}$, and weights $w_{1}, \ldots, w_{m}$, with $\mathbf{w}=\left(w_{1}, \ldots, w_{m}\right)$, and $v_{\mathbf{w}}:=w_{1}^{p / p_{1}} \ldots w_{m}^{p / p_{m}}$

$$
\mathcal{M}: L^{p_{1}}\left(w_{1}\right) \times \cdots \times L^{p_{m}}\left(w_{m}\right) \longrightarrow L^{p, \infty}\left(v_{\mathbf{w}}\right)
$$

if, and only if $\mathbf{w} \in A_{\mathbf{P}}$; that is, if

$$
[\mathbf{w}]_{A_{\mathbf{P}}}:=\sup _{Q}\left(f_{Q} v_{\mathbf{w}}\right)^{1 / p} \prod_{i=1}^{m}\left(f_{Q} w_{i}^{1-p_{i}^{\prime}}\right)^{1 / p_{i}^{\prime}}<\infty
$$

where $\left(f_{Q} w_{i}^{1-p_{i}^{\prime}}\right)^{1 / p_{i}^{\prime}}$ is replaced by $\left(\operatorname{ess} \inf _{x \in Q} w_{i}(x)\right)^{-1}$ if $p_{i}=1$. Moreover, if $1<p_{1}, \ldots, p_{m}<\infty$, then

$$
\mathcal{M}: L^{p_{1}}\left(w_{1}\right) \times \cdots \times L^{p_{m}}\left(w_{m}\right) \longrightarrow L^{p}\left(v_{\mathbf{w}}\right)
$$

if, and only if $\mathbf{w} \in A_{\mathbf{P}}$.

We are using throughout the paper the standard notation $T: X_{1} \times \cdots \times X_{m} \longrightarrow X_{0}$ to denote that $T$ is a bounded operator from $X_{1} \times \cdots \times X_{m}$ to $X_{0}$, where $X_{i}$ is an appropriate function space.

\subsection{Dyadic grids and sparse collections of cubes}

A general dyadic grid $\mathscr{D}$ is a collection of cubes in $\mathbb{R}^{n}$ with the following properties:

(a) For any $Q \in \mathscr{D}$, its side length $l_{Q}$ is of the form $2^{k}$, for some $k \in \mathbb{Z}$.

(b) For all $Q, R \in \mathscr{D}, Q \cap R \in\{\emptyset, Q, R\}$.

(c) The cubes of a fixed side length $2^{k}$ form a partition of $\mathbb{R}^{n}$.

The standard dyadic grid in $\mathbb{R}^{n}$ consists of the cubes $2^{-k}\left([0,1)^{n}+j\right)$, with $k \in \mathbb{Z}$ and $j \in \mathbb{Z}^{n}$. It is well-known (see [29]) that if one considers the perturbed dyadic grids

$$
\mathscr{D}_{\alpha}:=\left\{2^{-k}\left([0,1)^{n}+j+(-1)^{k} \alpha\right): k \in \mathbb{Z}, j \in \mathbb{Z}^{n}\right\},
$$

with $\alpha \in\left\{0, \frac{1}{3}\right\}^{n}$, then for any cube $Q \subseteq \mathbb{R}^{n}$, there exist $\alpha$ and a cube $Q_{\alpha} \in \mathscr{D}_{\alpha}$ such that $Q \subseteq Q_{\alpha}$ and $l_{Q_{\alpha}} \leq 6 l_{Q}$.

A collection of cubes $\mathcal{S}$ is said to be $\eta$-sparse if there exists $0<\eta<1$ such that for every cube $Q \in \mathcal{S}$, there exists a set $E_{Q} \subseteq Q$ with $\eta|Q| \leq\left|E_{Q}\right|$, and for every $Q \neq R \in \mathcal{S}, E_{R} \cap E_{Q}=\emptyset$.

For more information about these topics, see [34].

\subsection{Calderón-Zygmund operators}

We say that a function $\omega:[0, \infty) \rightarrow[0, \infty)$ is a modulus of continuity if it is continuous, increasing, sub-additive and such that $\omega(0)=0$. We say that $\omega$ satisfies 
the Dini condition if

$$
\|\omega\|_{\text {Dini }}:=\int_{0}^{1} \frac{\omega(t)}{t} d t<\infty .
$$

We give the definition of the multi-linear $\omega$-Calderón-Zygmund operators. We denote by $\mathscr{S}\left(\mathbb{R}^{n}\right)$ the space of all Schwartz functions on $\mathbb{R}^{n}$ and by $\mathscr{S}^{\prime}\left(\mathbb{R}^{n}\right)$ its dual space, the set of all tempered distributions on $\mathbb{R}^{n}$.

Definition 1 An $m$-linear $\omega$-Calderón-Zygmund operator is an $m$-linear and continuous operator $T: \mathscr{S}\left(\mathbb{R}^{n}\right) \times \cdots \times \mathscr{S}\left(\mathbb{R}^{n}\right) \longrightarrow \mathscr{S}^{\prime}\left(\mathbb{R}^{n}\right)$ for which there exists a locally integrable function $K\left(y_{0}, y_{1}, \ldots, y_{m}\right)$, defined away from the diagonal $y_{0}=y_{1}=\cdots=y_{m}$ in $\left(\mathbb{R}^{n}\right)^{m+1}$, satisfying, for some constant $C_{K}>0$, the size estimate

$$
\left|K\left(y_{0}, y_{1}, \ldots, y_{m}\right)\right| \leq \frac{C_{K}}{\left(\left|y_{0}-y_{1}\right|+\cdots+\left|y_{0}-y_{m}\right|\right)^{n m}}
$$

for all $\left(y_{0}, y_{1}, \ldots, y_{m}\right) \in\left(\mathbb{R}^{n}\right)^{m+1}$ with $y_{0} \neq y_{j}$ for some $j \in\{1, \ldots, m\}$, and the smoothness estimate

$$
\begin{aligned}
& \left|K\left(y_{0}, y_{1}, \ldots, y_{i}, \ldots, y_{m}\right)-K\left(y_{0}, y_{1}, \ldots, y_{i}^{\prime}, \ldots, y_{m}\right)\right| \\
& \leq \frac{C_{K}}{\left(\left|y_{0}-y_{1}\right|+\cdots+\left|y_{0}-y_{m}\right|\right)^{n m}} \omega\left(\frac{\left|y_{i}-y_{i}^{\prime}\right|}{\left(\left|y_{0}-y_{1}\right|+\cdots+\left|y_{0}-y_{m}\right|\right)^{n m}}\right),
\end{aligned}
$$

for $i=0, \ldots, m$ and whenever $\left|y_{i}-y_{i}^{\prime}\right| \leq \frac{1}{2} \max _{0 \leq j \leq m}\left|y_{i}-y_{j}\right|$, and such that

$$
T\left(f_{1}, \ldots, f_{m}\right)(x)=\int_{\mathbb{R}^{n}} \ldots \int_{\mathbb{R}^{n}} K\left(x, y_{1}, \ldots, y_{m}\right) f_{1}\left(y_{1}\right) \ldots f_{m}\left(y_{m}\right) d y_{1} \ldots d y_{m},
$$

whenever $f_{1}, \ldots, f_{m} \in \mathscr{C}_{c}^{\infty}\left(\mathbb{R}^{n}\right)$ and $x \notin \bigcap_{j=1}^{m}$ supp $f_{j}$, and for some exponents $1 \leq q_{1}, \ldots, q_{m}<\infty, T$ extends to a bounded $m$-linear operator from $L^{q_{1}}\left(\mathbb{R}^{n}\right) \times$ $\cdots \times L^{q_{m}}\left(\mathbb{R}^{n}\right)$ to $L^{q}\left(\mathbb{R}^{n}\right)$, with $\frac{1}{q}=\frac{1}{q_{1}}+\cdots+\frac{1}{q_{m}}$.

If we take $\omega(t)=t^{\varepsilon}$ for some $\varepsilon>0$, we recover the classical multi-linear CalderónZygmund operators. In general, an $m$-linear $\omega$-Calderón-Zygmund operator with $\omega$ satisfying the Dini condition can be extended to a bounded operator from $L^{1}\left(\mathbb{R}^{n}\right) \times$ $\cdots \times L^{1}\left(\mathbb{R}^{n}\right)$ to $L^{1 / m, \infty}\left(\mathbb{R}^{n}\right)$. The multi-linear Calderón-Zygmund theory has been investigated by many authors. For more information on this matter, see $[28,36,42]$ and the publications cited there.

\section{Sawyer-type inequalities for maximal operators}

We devote this section to the study of a novel restricted weak type inequality that extends the classical Sawyer-type inequality (1.1) for the Hardy-Littlewood maximal operator. To this end, we will need some previous results. 
The following lemma contains well-known results on weights (see $[19,21,26,40]$ ), but we will give most of their proofs since we need to keep track of the constants of the weights involved.

Lemma 1 Let $u$ and $w$ be weights.

(a) If $u \in A_{1}$, then $u^{-1} \in R H_{\infty}$, and $\left[u^{-1}\right]_{R H_{\infty}} \leq[u]_{A_{1}}$.

(b) If $u \in R H_{\infty}$, and $q>0$, then $u^{q} \in R H_{\infty}$. If $q \geq 1$, then $\left[u^{q}\right]_{R H_{\infty}} \leq[u]_{R H_{\infty}}^{q}$.

(c) If $u \in R H_{\infty}$, and $[u]_{R H_{\infty}} \leq \beta$, then there exists $r>1$, depending only on $n, \beta$, such that $u \in A_{r}$ and $[u]_{A_{r}} \leq c_{n, \beta}$. In particular, $R H_{\infty} \subseteq A_{\infty}$.

(d) If $u \in A_{\infty}$, and $w \in R H_{\infty}$, then $u w \in A_{\infty}$.

(e) If $u \in A_{1} \cap R H_{\infty}$, then $u \approx 1$.

Fix $p \geq 1$, and $f_{1}, \ldots, f_{m} \in L_{l o c}^{1}\left(\mathbb{R}^{n}\right)$, and let $v=\prod_{i=1}^{m}\left(M f_{i}\right)^{-1}$.

(f) $v^{p} \in R H_{\infty}$, and $1 \leq\left[v^{p}\right]_{R H_{\infty}} \leq c_{m, n, p}$.

(g) If $u \in A_{\infty}$, then $u v^{p} \in A_{\infty}$, with constant independent of $\mathbf{f}=\left(f_{1}, \ldots, f_{m}\right)$.

Proof To prove (a), fix a cube $Q \subseteq \mathbb{R}^{n}$. By Hölder's inequality, we have that

$$
|Q|=\int_{Q} u^{-1 / 2} u^{1 / 2} \leq\left(\int_{Q} u^{-1}\right)^{1 / 2}\left(\int_{Q} u\right)^{1 / 2},
$$

and hence,

$$
\left.\underset{x \in Q}{\operatorname{ess} \sup } u(x)^{-1}=\underset{x \in Q}{\operatorname{essinf}} u(x)\right)^{-1} \leq[u]_{A_{1}} \frac{|Q|}{u(Q)} \leq[u]_{A_{1}} f_{Q} u^{-1},
$$

and the desired result follows taking the supremum over all cubes $Q$.

The property (b) follows from [21, Theorem 4.2]. Let $q \geq 1$, and fix a cube $Q \subseteq \mathbb{R}^{n}$. Then,

$$
\underset{x \in Q}{\operatorname{ess} \sup } u(x) \leq[u]_{R H_{\infty}} f_{Q} u \leq[u]_{R H_{\infty}}\left(f_{Q} u^{q}\right)^{1 / q}
$$

from which the desired result follows, as before.

To prove (c), fix a cube $Q \subseteq \mathbb{R}^{n}$, and a measurable set $E \subseteq Q$. Then,

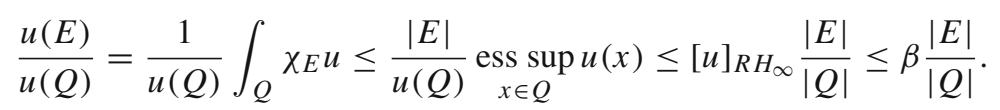

In particular, for every $\varepsilon>0$, and $\delta:=\frac{\varepsilon}{\beta}$, if $|E|<\delta|Q|$, then $u(E)<\varepsilon u(Q)$, and the desired result follows from this fact applying the last theorem in [46].

To prove (d), take $q, r>1$ such that $u \in A_{q}$ and $w \in A_{r}$. We will show that $u w \in A_{s}$, for $s:=q+r-1$. Fix a cube $Q \subseteq \mathbb{R}^{n}$. Then,

$$
f_{Q} u w \leq[w]_{R H_{\infty}}\left(f_{Q} u\right)\left(f_{Q} w\right)
$$


and in virtue of Hölder's inequality with exponent $\alpha:=1+\frac{r-1}{q-1}$,

$$
\begin{aligned}
\left(f_{Q}(u w)^{1-s^{\prime}}\right)^{s-1} & \leq\left(f_{Q} u^{\left(1-s^{\prime}\right) \alpha}\right)^{(s-1) / \alpha}\left(f_{Q} w^{\left(1-s^{\prime}\right) \alpha^{\prime}}\right)^{(s-1) / \alpha^{\prime}} \\
& =\left(f_{Q} u^{1-q^{\prime}}\right)^{q-1}\left(f_{Q} w^{1-r^{\prime}}\right)^{r-1}
\end{aligned}
$$

so $[u w]_{A_{s}} \leq[w]_{R H_{\infty}}[u]_{A_{q}}[w]_{A_{r}}<\infty$.

The property (e) follows immediately from Corollary 4.6 in [21].

To prove (f), observe that in virtue of [27, Theorem 7.2.7], we have that for $0<$ $\delta<1,\left(M f_{i}\right)^{\delta} \in A_{1}$, and $\left[\left(M f_{i}\right)^{\delta}\right]_{A_{1}} \leq \frac{c_{n}}{1-\delta}, i=1, \ldots, m$. In particular, $w:=$ $\prod_{i=1}^{m}\left(M f_{i}\right)^{\delta / m} \in A_{1}$, and $[w]_{A_{1}} \leq \prod_{i=1}^{m}\left[\left(M f_{i}\right)^{\delta}\right]_{A_{1}}^{1 / m} \leq \frac{c_{n}}{1-\delta}$. Since $v^{p}=w^{-m p / \delta}$, it follows from (a) and (b) that

$$
\left[v^{p}\right]_{R H_{\infty}} \leq\left[w^{-1}\right]_{R H_{\infty}}^{m p / \delta} \leq[w]_{A_{1}}^{m p / \delta} \leq\left(\frac{c_{n}}{1-\delta}\right)^{m p / \delta}
$$

so

$$
1 \leq\left[v^{p}\right]_{R H_{\infty}} \leq c_{m, n, p}:=\inf _{0<\delta<1}\left(\frac{c_{n}}{1-\delta}\right)^{m p / \delta} .
$$

To prove (g), we already know by (f) that $v^{p} \in R H_{\infty}$, with constant bounded by $c_{m, n, p}$, so by (c), there exists $r>1$, depending only on $m, n, p$, such that $\left[v^{p}\right]_{A_{r}} \leq$ $C_{m, n, p}$. By (d), for $q>1$ such that $u \in A_{q}$, and $s=s(m, n, p, q)=q+r-1$, $\left[u v^{p}\right]_{A_{s}} \leq \widetilde{C}_{m, n, p}[u]_{A_{q}}<\infty$.

The next lemma gives a result on weights that will be handy later on.

Lemma 2 Let $u$ and $v$ be weights, and suppose that $u \in A_{\infty}$. Then, $u v \in A_{\infty}$ if, and only if $v \in A_{\infty}(u)$.

Proof Let us first assume that $u v \in A_{\infty}$. Since $u \in A_{\infty}$, there exists $s>1$ such that $u \in R H_{s}$, and since $u v \in A_{\infty}$, there exists $r>1$ such that $u v \in A_{r}$. Take $q:=\frac{r s}{s-1}>1$. We will show that $v \in A_{q}(u)$. Fix a cube $Q$. Then,

$$
\begin{aligned}
I_{Q} & :=\left(\frac{1}{u(Q)} \int_{Q} v u\right)\left(\frac{1}{u(Q)} \int_{Q} v^{1-q^{\prime}} u\right)^{q-1} \\
& =\left(\frac{|Q|}{u(Q)}\right)^{q}\left(\frac{1}{|Q|} \int_{Q} v u\right)\left(\frac{1}{|Q|} \int_{Q}(v u)^{1-q^{\prime}} u^{q^{\prime}}\right)^{q-1} .
\end{aligned}
$$


Take $\alpha:=\frac{q-1}{r-1}=1+\frac{r}{(r-1)(s-1)}>1$ and observe that $\left(1-q^{\prime}\right) \alpha=1-r^{\prime}, \frac{q-1}{\alpha}=r-1$, $q^{\prime} \alpha^{\prime}=s$, and $\frac{q-1}{\alpha^{\prime}}=\frac{q}{s}$. Using Hölder's inequality with exponent $\alpha$, we get that

$$
\begin{aligned}
\left(\frac{1}{|Q|} \int_{Q}(v u)^{1-q^{\prime}} u^{q^{\prime}}\right)^{q-1} & \leq\left(\frac{1}{|Q|} \int_{Q}(v u)^{\left(1-q^{\prime}\right) \alpha}\right)^{\frac{q-1}{\alpha}}\left(\frac{1}{|Q|} \int_{Q} u^{q^{\prime} \alpha^{\prime}}\right)^{\frac{q-1}{\alpha^{\prime}}} \\
& =\left(\frac{1}{|Q|} \int_{Q}(v u)^{1-r^{\prime}}\right)^{r-1}\left(\frac{1}{|Q|} \int_{Q} u^{s}\right)^{q / s} \\
& \leq[u]_{R H_{s}}^{q}\left(\frac{1}{|Q|} \int_{Q}(v u)^{1-r^{\prime}}\right)^{r-1}\left(\frac{u(Q)}{|Q|}\right)^{q}
\end{aligned}
$$

Hence,

$$
I_{Q} \leq[u]_{R H_{s}}^{q}\left(\frac{1}{|Q|} \int_{Q} v u\right)\left(\frac{1}{|Q|} \int_{Q}(v u)^{1-r^{\prime}}\right)^{r-1} \leq[u]_{R H_{s}}^{q}[u v]_{A_{r}},
$$

and $[v]_{A_{q}(u)}=\sup _{Q} I_{Q} \leq[u]_{R H_{s}}^{q}[u v]_{A_{r}}<\infty$.

For the converse, let us assume that $v \in A_{\infty}(u)$. It follows from Theorem 3.1 in [23] that there exist $\delta, C>0$ such that for every cube $Q \subseteq \mathbb{R}^{n}$ and every measurable set $E \subseteq Q$,

$$
\frac{u(E)}{u(Q)} \leq C\left(\frac{u v(E)}{u v(Q)}\right)^{\delta}
$$

Similarly, since $u \in A_{\infty}$, there exist $\varepsilon, c>0$ such that for every cube $Q \subseteq \mathbb{R}^{n}$ and every measurable set $E \subseteq Q$,

$$
\frac{|E|}{|Q|} \leq c\left(\frac{u(E)}{u(Q)}\right)^{\varepsilon}
$$

so for every cube $Q \subseteq \mathbb{R}^{n}$ and every measurable set $E \subseteq Q$,

$$
\frac{|E|}{|Q|} \leq c C^{\varepsilon}\left(\frac{u v(E)}{u v(Q)}\right)^{\varepsilon \delta}
$$

and hence, $u v \in A_{\infty}$.

Remark 1 This result is an extension of Lemma 2.1 in [19], where it is shown that if $u \in A_{1}$ and $v \in A_{\infty}(u)$, then $u v \in A_{\infty}$.

We introduce a weighted version of the dyadic Hardy-Littlewood maximal operator.

Definition 2 Let $\mathscr{D}$ be a general dyadic grid in $\mathbb{R}^{n}$, and let $u$ be a weight. For a measurable function $f$, we consider the function

$$
M_{u}^{\mathscr{D}} f(x):=\sup _{\mathscr{D} \ni Q \ni x} \frac{1}{u(Q)} \int_{Q}|f(y)| u(y) d y, \quad x \in \mathbb{R}^{n},
$$


where the supremum is taken over all cubes $Q \in \mathscr{D}$ that contain $x$. If $u=1$, we simply write $M^{\mathscr{D}} f$.

The following bound for the operator $M_{u}^{\mathscr{D}}$ is essential.

Theorem 1 Let $\mathscr{D}$ be a general dyadic grid in $\mathbb{R}^{n}$, and let $u$ and $v$ be weights. If $u \in A_{\infty}$ and $u v \in A_{\infty}$, then there exists a constant $C_{u, v}$, independent of $\mathscr{D}$, such that for every measurable function $f$,

$$
\left\|\frac{M_{u}^{\mathscr{D}}(f v)}{v}\right\|_{L^{1, \infty}(u v)} \leq C_{u, v} \int_{\mathbb{R}^{n}}|f(x)| u(x) v(x) d x .
$$

Proof In virtue of Lemma 2, $v \in A_{\infty}(u)$ and hence, this theorem follows from the proof of Theorem 1.4 in [19].

Remark 2 If we examine the proof of Theorem 1.4 in [19], and we combine it with Appendix A in [20], we can take

$$
C_{u, v}=2^{q}\left(2^{n} r[u v]_{A_{r}^{\mathcal{R}}}\right)^{r(q-1)}\left\|M_{u}\right\|_{L^{q}\left(u v^{1-q}\right)}^{q},
$$

where $r, q>1$ are such that $u v \in A_{r}^{\mathcal{R}}$ and $v \in A_{q^{\prime}}(u)$.

Remark 3 The bound of Theorem 1 also holds for the weighted Hardy-Littlewood maximal operator $M_{u}$, with constant

$$
C:=2^{n} 6^{n p} p^{p}[u]_{A_{p}^{\mathcal{R}}}^{p} C_{u, v}
$$

where $p \geq 1$ is such that $u \in A_{p}^{\mathcal{R}}$.

We can now state and prove the main result of this section.

Theorem 2 Fix $p \geq 1$, and let $u$ and $v$ be weights such that $u \in A_{p}^{\mathcal{R}}$ and $u v^{p} \in A_{\infty}$. Then, there exists a constant $C>0$ such that for every measurable function $f$,

$$
\left\|\frac{M f}{v}\right\|_{L^{p, \infty}\left(u v^{p}\right)} \leq C\|f\|_{L^{p, 1}(u)} .
$$

Proof It is known (see $[29,32])$ that there exists a collection $\left\{\mathscr{D}_{\alpha}\right\}_{\alpha}$ of $2^{n}$ general dyadic grids in $\mathbb{R}^{n}$ such that

$$
M f \leq 6^{n} \sum_{\alpha=1}^{2^{n}} M^{\mathscr{D}_{\alpha}} f
$$

Hence,

$$
\left\|\frac{M f}{v}\right\|_{L^{p, \infty}\left(u v^{p}\right)} \leq 12^{n} \sum_{\alpha=1}^{2^{n}}\left\|\frac{M^{\mathscr{D}_{\alpha}} f}{v}\right\|_{L^{p, \infty}\left(u v^{p}\right)},
$$


and it suffices to establish the result for the operator $M^{\mathscr{D}}$, with $\mathscr{D}$ a general dyadic grid in $\mathbb{R}^{n}$.

We first discuss the case $p=1$, which was proved in [19]. We reproduce the proof here keeping track of the constants. By the definition of the $A_{1}$ condition,

$$
\frac{1}{|Q|} \int_{Q}|f| \leq[u]_{A_{1}} \frac{1}{u(Q)} \int_{Q}|f| u,
$$

for every cube $Q \in \mathscr{D}$, so we get that $M^{\mathscr{D}} f \leq[u]_{A_{1}} M_{u}^{\mathscr{D}} f$. This estimate combined with Theorem 1 gives that

$$
\left\|\frac{M^{\mathscr{D}} f}{v}\right\|_{L^{1, \infty}(u v)} \leq[u]_{A_{1}}\left\|\frac{M_{u}^{\mathscr{D}}(v f / v)}{v}\right\|_{L^{1, \infty}(u v)} \leq[u]_{A_{1}} C_{u, v} \int_{\mathbb{R}^{n}}|f| u,
$$

and hence, the desired result follows, with $C=24^{n}[u]_{A_{1}} C_{u, v}$.

Now, we discuss the case $p>1$. Let us take $f=\chi_{E}$, with $E$ a measurable set in $\mathbb{R}^{n}$, and fix a cube $Q \in \mathscr{D}$. As before, by the definition of the $A_{p}^{\mathcal{R}}$ condition,

$$
\frac{1}{|Q|} \int_{Q} f \leq\|u\|_{A_{p}^{\mathcal{R}}}\left(\frac{u(E \cap Q)}{u(Q)}\right)^{1 / p}
$$

so we get that $M^{\mathscr{D}}\left(\chi_{E}\right) \leq p[u]_{A_{p}^{\mathcal{R}}}\left(M_{u}^{\mathscr{D}}\left(\chi_{E}\right)\right)^{1 / p}$. In particular,

$$
\left\|\frac{M^{\mathscr{D}}\left(\chi_{E}\right)}{v}\right\|_{L^{p, \infty}\left(u v^{p}\right)} \leq p[u]_{A_{p}^{\mathcal{R}}}\left\|\frac{M_{u}^{\mathscr{D}}\left(\chi_{E}\right)}{v^{p}}\right\|_{L^{1, \infty}\left(u v^{p}\right)}^{1 / p} .
$$

We can now apply Theorem 1 to conclude that

$$
\left\|\frac{M_{u}^{\mathscr{D}}\left(\chi_{E}\right)}{v^{p}}\right\|_{L^{1, \infty}\left(u v^{p}\right)}=\left\|\frac{M_{u}^{\mathscr{D}}\left(v^{p} \chi_{E} / v^{p}\right)}{v^{p}}\right\|_{L^{1, \infty\left(u v^{p}\right)}} \leq C_{u, v^{p}} u(E) .
$$

Combining all the previous estimates, we have that

$$
\left\|\frac{M\left(\chi_{E}\right)}{v}\right\|_{L^{p, \infty\left(u v^{p}\right)}} \leq 24^{n}[u]_{A_{p}^{\mathcal{R}}} C_{u, v^{p}}^{1 / p}\left\|\chi_{E}\right\|_{L^{p, 1}(u)} .
$$

Since $p>1, L^{p, \infty}\left(u v^{p}\right)$ is a Banach space, and by standard arguments (see [27, Exercise 1.4.7]), we can extend the previous estimate to arbitrary measurable functions $f$, gaining a factor of $4 p^{\prime}$ in the constant. Hence, the desired result follows, with $C=4 \cdot 24^{n} p^{\prime}[u]_{A_{p}^{\mathcal{R}}} C_{u, v^{p}}^{1 / p}$.

Remark 4 For $p=1$ and $u \in A_{1}$, a more general version of Theorem 2 was established in [39], replacing the hypothesis that $u v \in A_{\infty}$ by the weaker assumption that $v \in A_{\infty}$. 
It is unknown to us whether the hypothesis that $u v^{p} \in A_{\infty}$ can be replaced by $v \in A_{\infty}$ when $p>1$.

In virtue of Lemma 1 , if $u \in A_{\infty}$ and $v \in R H_{\infty}$, then for every $p \geq 1, u v^{p} \in A_{\infty}$, and we have a whole class of non-trivial examples of weights that satisfy the hypotheses of Theorem 2.

Observe that the conclusion of Theorem 2 is completely elementary if $p>1$ and $u \in A_{p}$, since

$$
\begin{aligned}
\left\|\frac{M f}{v}\right\|_{L^{p, \infty}\left(u v^{p}\right)} & \leq\left\|\frac{M f}{v}\right\|_{L^{p}\left(u v^{p}\right)} \\
& =\|M f\|_{L^{p}(u)} \lesssim[u]_{A_{p}}^{\frac{1}{p-1}}\|f\|_{L^{p}(u)} \lesssim[u]_{A_{p}}^{\frac{1}{p-1}}\|f\|_{L^{p, 1}(u)} .
\end{aligned}
$$

However, this argument doesn't work in the general case, because the inequality

$$
\left\|\frac{h}{v}\right\|_{L^{p, \infty}\left(u v^{p}\right)} \lesssim\|h\|_{L^{p, \infty}(u)}
$$

may fail for some measurable functions $h$ on $\mathbb{R}^{n}$, and arbitrary weights $u$ and $v$, as can be seen by choosing $h(x)=|x|^{-\frac{n}{p}} \chi_{\left\{y \in \mathbb{R}^{n}:|y| \geq 1\right\}}(x), u=1$, and $v(x)=$ $h(x)+\chi_{\left\{y \in \mathbb{R}^{n}:|y|<1\right\}}(x)$, with $0<p<\infty$.

To provide applications of Theorem 2 we need to give a more precise estimate of the constant $C$ that appears there in terms of the corresponding constants of the weights involved. We achieve this in the following lemma.

Lemma 3 In Theorem 2, if $r \geq 1$ is such that $u v^{p} \in A_{r}^{\mathcal{R}}$, then one can take

$$
C=\mathscr{E}_{r, p}^{n}\left([u]_{A_{p}^{\mathcal{R}}},\left[u v^{p}\right]_{A_{r}^{\mathcal{R}}}\right)
$$

where $\mathscr{E}_{r, p}:[1, \infty)^{2} \longrightarrow(0, \infty)$ is a function that increases in each variable, and it depends only on $r, p$, and the dimension $n$.

Proof We first discuss the case when $r>1$. We already know that we can take

$$
C= \begin{cases}24^{n}[u]_{A_{1}} C_{u, v}, & p=1 \\ 4 \cdot 24^{n} p^{\prime}[u]_{A_{p}^{\mathcal{R}}} C_{u, v}^{1 / p}, & p>1\end{cases}
$$

and in virtue of Remark 2,

$$
C_{u, v^{p}}=2^{q}\left(2^{n} r\left[u v^{p}\right]_{A_{r}^{\mathcal{R}}}\right)^{r(q-1)}\left\|M_{u}\right\|_{L^{q}\left(u v^{p(1-q)}\right)}^{q},
$$

where $r, q>1$ are such that $u v^{p} \in A_{r}^{\mathcal{R}}$ and $v^{p} \in A_{q^{\prime}}(u)$. For convenience, we write $V:=v^{p}$. Let us first bound the factor $\left\|M_{u}\right\|_{L^{q}\left(u V^{1-q}\right)}^{q}$. For the space of homogeneous 
type $\left(\mathbb{R}^{n}, d_{\infty}, u(x) d x\right)$, it follows from the proof of Theorem 1.3 in [30] that

$$
\left\|M_{u}\right\|_{L^{q}\left(u V^{1-q}\right)}^{q} \leq 2^{q-1} q^{\prime} 40^{q D_{u}}\left(1+6 \cdot 800^{D_{u}}\right)[V]_{A_{\infty}(u)}[V]_{A_{q^{\prime}}(u)}^{q-1},
$$

where $D_{u}:=p \log _{2}\left(2^{n} p[u]_{A} \mathcal{R}\right)$. Now, given a cube $Q \subseteq \mathbb{R}^{n}$, and applying Hölder's inequality with exponent $q$, we have that

$$
\begin{aligned}
\int_{Q} M_{u}\left(V \chi_{Q}\right) u & =\int_{Q} \frac{M_{u}\left(V \chi_{Q}\right)}{V} u V \leq\left\|\frac{M_{u}\left(V \chi_{Q}\right)}{V}\right\|_{L^{q}(u V)} u V(Q)^{1 / q^{\prime}} \\
& =\left\|M_{u}\left(V \chi_{Q}\right)\right\|_{L^{q}\left(u V^{1-q}\right)} u V(Q)^{1 / q^{\prime}} \\
& \leq\left\|M_{u}\right\|_{L^{q}\left(u V^{1-q}\right)}\left\|V \chi_{Q}\right\|_{L^{q}\left(u V^{1-q}\right)} u V(Q)^{1 / q^{\prime}} \\
& =\left\|M_{u}\right\|_{L^{q}\left(u V^{1-q}\right)} u V(Q)
\end{aligned}
$$

and taking the supremum over all cubes $Q$, we get that

$$
[V]_{A_{\infty}(u)} \leq\left\|M_{u}\right\|_{L^{q}\left(u V^{1-q}\right)} .
$$

Combining the previous estimates, we obtain that

$$
\left\|M_{u}\right\|_{L^{q}\left(u V^{1-q}\right)}^{q} \leq\left(2^{q-1} q^{\prime} 40^{q D_{u}}\left(1+6 \cdot 800^{D_{u}}\right)\right)^{q^{\prime}}[V]_{A_{q^{\prime}}(u)}^{q} .
$$

Now, we will bound the factor $[V]_{A_{q^{\prime}}(u)}^{q}$. In virtue of [29, Proposition 2.2], and using the definitions of $[u]_{A_{2 p}}$ and $[u]_{A_{p}^{\mathcal{R}}}$, and Kolmogorov's inequalities, we can deduce that

$$
[u]_{A_{\infty}} \leq c_{n}[u]_{A_{2 p}} \leq(2 p-1)^{2 p-1} c_{n}[u]_{A_{p}^{\mathcal{R}}}^{2 p}=: c_{p, n}[u]_{A_{p}^{\mathcal{R}}}^{2 p}
$$

and applying Theorem 2.3 in [30], $u \in R H_{S}$ for $s=1+\frac{1}{2^{n+1} c_{p, n}[u]_{A_{p}^{R}}^{2 p}-1}$, and $[u]_{R H_{s}} \leq$ 2. Since $u V \in A_{2 r}$, Lemma 2 tells us that if we choose $q^{\prime}=2 r s^{\prime}$, then

$$
[V]_{A_{q^{\prime}}(u)}^{q} \leq[u]_{R H_{s}}^{q q^{\prime}}[u V]_{A_{2 r}}^{q} \leq 2^{q q^{\prime}}(2 r-1)^{q(2 r-1)}[u V]_{A_{r}^{\mathcal{R}}}^{2 r q}
$$

Finally, observe that $q^{\prime}=2^{n+2} r c_{p, n}[u]_{A_{p}^{\mathcal{R}}}^{2 p}$, and $1<q \leq 2$, so

$$
\begin{aligned}
C_{u, V} & \leq 2^{2}\left(2^{n} r[u V]_{A_{r}^{\mathcal{R}}}\right)^{r} \times\left(2 q^{\prime} 40^{2 D_{u}}\left(1+6 \cdot 800^{D_{u}}\right)\right)^{q^{\prime}} \\
& \times 2^{2 q^{\prime}}(2 r-1)^{4 r-2}[u V]_{A_{r}^{\mathcal{R}}}^{4 r} \\
& \leq 2^{2+n r}(2 r-1)^{4 r-2} r^{r}\left[u v^{p}\right]_{A_{r}^{\mathcal{R}}}^{5 r} \\
& \times\left(2^{n+5} r c_{p, n}[u]_{A_{p}^{\mathcal{R}}}^{2 p} 40^{5 p \log _{2}\left(2^{n} p[u]_{A_{p}^{\mathcal{R}}}\right)}\right)^{2^{n+2} r c_{p, n}[u]_{A_{p}^{\mathcal{R}}}^{2 p}}
\end{aligned}
$$




$$
=: C_{r, p}^{n}\left([u]_{A_{p}^{\mathcal{R}}},\left[u v^{p}\right]_{A_{r}^{\mathcal{R}}}\right)
$$

and the desired result follows, with

$$
\mathscr{E}_{r, p}\left([u]_{A_{p}^{\mathcal{R}}},\left[u v^{p}\right]_{A_{r}^{\mathcal{R}}}\right)= \begin{cases}24^{n}[u]_{A_{1}} C_{r, 1}^{n}\left([u]_{A_{1}},[u v]_{A_{r}^{\mathcal{R}}}\right), & p=1, \\ 4 \cdot 24^{n} p^{\prime}[u]_{A_{p}^{\mathcal{R}}} C_{r, p}^{n}\left([u]_{A_{p}^{\mathcal{R}}},\left[u v^{p}\right]_{A_{r}^{\mathcal{R}}}\right)^{1 / p}, & p>1 .\end{cases}
$$

The case when $r=1$ follows, for example, from the case when $r=2$ and the fact that if $u v^{p} \in A_{1}$, then $\left[u v^{p}\right]_{A_{2}^{\mathcal{R}}} \leq\left[u v^{p}\right]_{A_{2}}^{1 / 2} \leq\left[u v^{p}\right]_{A_{1}}^{1 / 2}$.

Remark 5 It would be interesting to obtain the sharp dependence of $C$ on the constants of the weights involved; our results are most certainly far from optimal.

\section{Applications}

In this section, we will provide several applications of the Sawyer-type inequality established in Theorem 2, obtaining mixed restricted weak type estimates for multivariable maximal operators, sparse operators and Calderón-Zygmund operators.

The first result that we present is the converse of Theorem 3.3 in [14], that was left as an open question. Combining both theorems, we obtain the complete characterization of the restricted weak type bounds of the operator $M^{\otimes}$ for $A_{\infty}$ weights.

Theorem 3 Let $1 \leq p_{1}, \ldots, p_{m}<\infty$, and let $\frac{1}{p}=\frac{1}{p_{1}}+\cdots+\frac{1}{p_{m}}$. Let $w_{1}, \ldots, w_{m}$ be weights, with $w_{i} \in A_{p_{i}}^{\mathcal{R}}, i=1, \ldots, m$. Then, there exists a constant $C>0$ such that the inequality

$$
\left\|M^{\otimes}(\mathbf{f})\right\|_{L^{p, \infty}\left(v_{\mathbf{w}}\right)} \leq C \prod_{i=1}^{m}\left\|f_{i}\right\|_{L^{p_{i}, 1}\left(w_{i}\right)}
$$

holds for every vector of measurable functions $\mathbf{f}=\left(f_{1}, \ldots, f_{m}\right)$.

Proof The case when $p_{1}=\cdots=p_{m}=1$ was proved in [36], and we build upon that proof to demonstrate the remaining cases.

We can assume, without loss of generality, that $f_{i} \in L_{c}^{\infty}\left(\mathbb{R}^{n}\right), i=1, \ldots, m$. Fix $t>0$ and define

$$
E_{t}:=\left\{x \in \mathbb{R}^{n}: t<M^{\otimes}(\mathbf{f})(x) \leq 2 t\right\} .
$$

For $i=1, \ldots, m$, and taking $\tilde{v}_{i}:=\prod_{j \neq i}\left(M f_{j}\right)^{-1}$, we have that

$$
E_{t}=\left\{x \in \mathbb{R}^{n}: t \tilde{v}_{i}(x)<M f_{i}(x) \leq 2 t \tilde{v}_{i}(x)\right\} .
$$


Using the fact that $\tilde{v}_{i} \in R H_{\infty}$, with constant independent of $\mathbf{f}$ (see Lemma 1), Hölder's inequality, and Theorem 2, we obtain that

$$
\begin{aligned}
\lambda_{M^{\otimes}(\mathbf{f})}^{v_{\mathbf{w}}}(t)-\lambda_{M^{\otimes}(\mathbf{f})}^{v_{\mathbf{w}}}(2 t) & =\int_{E_{t}} v_{\mathbf{w}} \leq \int_{E_{t}}\left(\frac{M^{\otimes}(\mathbf{f})}{t}\right)^{p} v_{\mathbf{w}} \\
& \leq \frac{1}{t^{p}} \prod_{i=1}^{m}\left(\int_{E_{t}}\left(M f_{i}\right)^{p_{i}} w_{i}\right)^{p / p_{i}} \\
& \leq 2^{m p} t^{(m-1) p} \prod_{i=1}^{m}\left(\int_{\left\{\frac{M f_{i}}{\hat{v}_{i}}>t\right\}} \tilde{v}_{i}^{p_{i}} w_{i}\right)^{p / p_{i}} \\
& \leq 2^{m p} C_{1}^{p} \ldots C_{m}^{p} \frac{1}{t^{p}} \prod_{i=1}^{m}\left\|f_{i}\right\|_{L^{p_{i}, 1}\left(w_{i}\right)}^{p}
\end{aligned}
$$

Iterating this result, we get that for each $t>0$ and every natural number $N$,

$$
\lambda_{M^{\otimes}(\mathbf{f})}^{\nu_{\mathbf{w}}}(t) \leq 2^{m p} C_{1}^{p} \ldots C_{m}^{p}\left(\sum_{j=0}^{N} \frac{1}{2^{j p}}\right) \frac{1}{t^{p}} \prod_{i=1}^{m}\left\|f_{i}\right\|_{L^{p_{i}, 1}\left(w_{i}\right)}^{p}+\lambda_{M^{\otimes}(\mathbf{f})}^{v_{\mathbf{w}}}\left(2^{N+1} t\right),
$$

and letting $N$ tend to infinity, the last term vanishes, and we conclude that

$$
\lambda_{M^{\otimes}(\mathbf{f})}^{v_{\mathbf{w}}}(t) \leq \frac{2^{(m+1) p}}{2^{p}-1} C_{1}^{p} \ldots C_{m}^{p} \frac{1}{t^{p}} \prod_{i=1}^{m}\left\|f_{i}\right\|_{L^{p_{i}, 1}\left(w_{i}\right)}^{p} .
$$

Observe that in virtue of Lemma 1 , for $i=1, \ldots, m$, we have that $w_{i} \tilde{v}_{i}^{p_{i}} \in A_{s_{i}}$, where $s_{i}>1$ depends only on $m, n, p_{i}$, and

$$
\left[w_{i} \tilde{v}_{i}^{p_{i}}\right]_{A_{s_{i}}^{\mathcal{R}}}^{s_{i}} \leq\left[w_{i} \tilde{v}_{i}^{p_{i}}\right]_{A_{s_{i}}} \lesssim m, n, p_{i}\left[w_{i}\right]_{A_{2 p_{i}}} \lesssim_{m, n, p_{i}}\left[w_{i}\right]_{A_{p_{i}}^{\mathcal{R}}}^{2 p_{i}}
$$

so by Lemma 3, we have that $C_{i} \leq \mathscr{E}_{s_{i}, p_{i}}\left(\left[w_{i}\right]_{A_{p_{i}}^{\mathcal{R}}}, C_{m, n, p_{i}}\left[w_{i}\right]_{A_{p_{i}}^{\mathcal{R}}}^{2 p_{i} / s_{i}}\right)$, and hence, the desired result follows, with

$$
C=\frac{2^{m+1}}{\left(2^{p}-1\right)^{1 / p}} \prod_{i=1}^{m} \mathscr{E}_{S_{i}, p_{i}}\left(\left[w_{i}\right]_{A_{p_{i}}^{\mathcal{R}}}, C_{m, n, p_{i}}\left[w_{i}\right]_{A_{p_{i}}^{\mathcal{R}}}^{2 p_{i} / s_{i}}\right)
$$

which depends on the constants of the weights $w_{1}, \ldots, w_{m}$ in an increasing way.

The next application that we provide is an extension of Theorem 2 to the multivariable setting, which in turn, extends Theorem 3 . The proof is based on the previous one, and is similar to that of Theorem 1.4 in [40]. 
Theorem 4 Let $1 \leq p_{1}, \ldots, p_{m}<\infty$, and let $\frac{1}{p}=\frac{1}{p_{1}}+\cdots+\frac{1}{p_{m}}$. Let $w_{1}, \ldots, w_{m}$ be weights, with $w_{i} \in A_{p_{i}}^{\mathcal{R}}, i=1, \ldots, m$. Let $v$ be a weight such that $v_{\mathbf{w}} v^{p}$ is a weight, and $w_{i} v^{p_{i}} \in A_{\infty}, i=1, \ldots, m$. Then, there exists a constant $C>0$ such that the inequalities

$$
\left\|\frac{\mathcal{M}(\mathbf{f})}{v}\right\|_{L^{p, \infty}\left(v_{\mathbf{w}} v^{p}\right)} \leq\left\|\frac{M^{\otimes}(\mathbf{f})}{v}\right\|_{L^{p, \infty}\left(v_{\mathbf{w}} v^{p}\right)} \leq C \prod_{i=1}^{m}\left\|f_{i}\right\|_{L^{p_{i}, 1}\left(w_{i}\right)}
$$

hold for every vector of measurable functions $\mathbf{f}=\left(f_{1}, \ldots, f_{m}\right)$.

Proof The first inequality follows from the fact that $\mathcal{M}(\mathbf{f}) \leq M^{\otimes}(\mathbf{f})$. For the second one, we can assume, without loss of generality, that $f_{i} \in L_{c}^{\infty}\left(\mathbb{R}^{n}\right), i=1, \ldots, m$. Fix $y, R>0$ and define

$$
E_{y}^{R}:=\left\{x \in \mathbb{R}^{n}:|x|<R, y v(x)<M^{\otimes}(\mathbf{f})(x) \leq 2 y v(x)\right\} .
$$

For $i=1, \ldots, m$, and taking $\tilde{v}_{i}:=\prod_{j \neq i}\left(M f_{j}\right)^{-1}$, and $v_{i}:=\tilde{v}_{i} v$, we have that

$$
E_{y}^{R}=\left\{x \in \mathbb{R}^{n}:|x|<R, y v_{i}(x)<M f_{i}(x) \leq 2 y v_{i}(x)\right\}
$$

Since $\tilde{v}_{i} \in R H_{\infty}$, and $w_{i} v^{p_{i}} \in A_{\infty}$, we have that $w_{i} v_{i}^{p_{i}} \in A_{\infty}$, with constant independent of $\mathbf{f}$ (see Lemma 1). In virtue of Hölder's inequality and Theorem 2, we get that

$$
\begin{aligned}
& v_{\mathbf{w}} v^{p}\left(\left\{x \in \mathbb{R}^{n}:|x|<R, \frac{M^{\otimes}(\mathbf{f})(x)}{v(x)}>y\right\}\right) \\
& -v_{\mathbf{w}} v^{p}\left(\left\{x \in \mathbb{R}^{n}:|x|<R, \frac{M^{\otimes}(\mathbf{f})(x)}{v(x)}>2 y\right\}\right) \\
& =\int_{E_{y}^{R}} v_{\mathbf{w}} v^{p} \leq \int_{E_{y}^{R}}\left(\frac{M^{\otimes}(\mathbf{f})}{y}\right)^{p} v_{\mathbf{w}} \leq \frac{1}{y^{p}} \prod_{i=1}^{m}\left(\int_{E_{y}^{R}}\left(M f_{i}\right)^{p_{i}} w_{i}\right)^{p / p_{i}} \\
& \leq 2^{m p} y^{(m-1) p} \prod_{i=1}^{m}\left(\int_{\left\{\frac{M f_{i}}{v_{i}}>y\right\}} v_{i}^{p_{i}} w_{i}\right)^{p / p_{i}} \leq 2^{m p} C_{1}^{p} \ldots C_{m}^{p} \frac{1}{y^{p}} \prod_{i=1}^{m}\left\|f_{i}\right\|_{L^{p}, 1}^{p}\left(w_{i}\right)
\end{aligned}
$$

Iterating this result, we deduce that for each $y>0$ and every natural number $N$,

$$
\begin{aligned}
& v_{\mathbf{w}} v^{p}\left(\left\{x \in \mathbb{R}^{n}:|x|<R, \frac{M^{\otimes}(\mathbf{f})(x)}{v(x)}>y\right\}\right) \\
& \leq 2^{m p} C_{1}^{p} \ldots C_{m}^{p}\left(\sum_{j=0}^{N} \frac{1}{2^{j p}}\right) \frac{1}{y^{p}} \prod_{i=1}^{m}\left\|f_{i}\right\|_{L^{p_{i}, 1}\left(w_{i}\right)}^{p} \\
& +v_{\mathbf{w}} v^{p}\left(\left\{x \in \mathbb{R}^{n}:|x|<R, \frac{M^{\otimes}(\mathbf{f})(x)}{v(x)}>2^{N+1} y\right\}\right),
\end{aligned}
$$


and letting first $N$ tend to infinity, and then $R$, the last term vanishes, and we conclude that

$$
\lambda_{\frac{M^{\otimes}(\mathbf{f})}{v}}^{v_{\mathbf{w}} v^{p}}(y) \leq \frac{2^{(m+1) p}}{2^{p}-1} C_{1}^{p} \ldots C_{m}^{p} \frac{1}{y^{p}} \prod_{i=1}^{m}\left\|f_{i}\right\|_{L^{p_{i}, 1}\left(w_{i}\right)}^{p} .
$$

For $i=1, \ldots, m$, if we take $q_{i}>1$ such that $w_{i} v^{p_{i}} \in A_{q_{i}}^{\mathcal{R}}$, in virtue of Lemma 1 , we have that $w_{i} v_{i}^{p_{i}} \in A_{s_{i}}$, where $s_{i}>1$ depends only on $m, n, p_{i}, q_{i}$, and $\left[w_{i} v_{i}^{p_{i}}\right]_{A_{s_{i}}^{\mathcal{R}}}^{s_{i}} \lesssim_{m, n, p_{i}, q_{i}}\left[w_{i} v^{p_{i}}\right]_{A_{q_{i}}^{\mathcal{R}}}^{2 q_{i}}$, so by Lemma 3 , we have that $C_{i} \leq$ $\mathscr{E}_{s_{i}, p_{i}}\left(\left[w_{i}\right]_{A_{p_{i}}^{\mathcal{R}}}, C_{m, n, p_{i}, q_{i}}\left[w_{i} v^{p_{i}}\right]_{A_{q_{i}}^{\mathcal{R}}}^{2 q_{i} / s_{i}}\right)$, and hence, the desired result follows, with

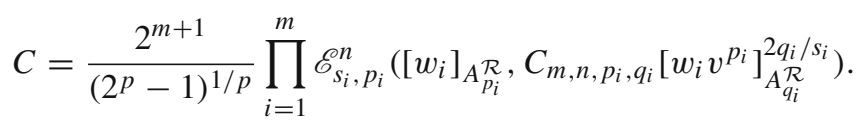

Remark 6 In the case when $p_{1}=\cdots=p_{m}=1$, the previous result is a corollary of Theorem 1.4 in [40].

Observe that if we take weights $w_{i} \in A_{p_{i}}^{\mathcal{R}}, i=1, \ldots, m$, and $v \in R H_{\infty}$, then the hypotheses of Theorem 4 are satisfied.

The next result will be crucial to work with Calderón-Zygmund operators in the mixed restricted weak setting.

Theorem 5 Let $0<p<\infty$, let $\mathcal{S}$ be an $\eta$-sparse collection of cubes, and let $v, w$ be weights. Suppose that there exists $0<\varepsilon \leq 1$ such that $\varepsilon<p, w v^{-\varepsilon} \in A_{\infty}$, and

$$
\left[v^{-\varepsilon}\right]_{R H_{\infty}(w)}:=\sup _{Q} \frac{w(Q)}{w v^{-\varepsilon}(Q)}\left\|\chi Q v^{-\varepsilon}\right\|_{L^{\infty}(w)}<\infty .
$$

Then, there exists a constant $C>0$, independent of $\mathcal{S}$, such that the inequality

$$
\left\|\frac{\mathcal{A}_{\mathcal{S}}(\mathbf{f})}{v}\right\|_{L^{p, \infty}(w)} \leq C\left\|\frac{\mathcal{M}(\mathbf{f})}{v}\right\|_{L^{p, \infty}(w)}
$$

holds for every vector of measurable functions $\mathbf{f}=\left(f_{1}, \ldots, f_{m}\right)$.

Proof In virtue of Kolmogorov's inequalities, we obtain that

$$
\left\|\frac{\mathcal{A}_{\mathcal{S}}(\mathbf{f})}{v}\right\|_{L^{p, \infty}(w)} \leq \sup _{0<w(F)<\infty}\left\|\frac{\mathcal{A}_{\mathcal{S}}(\mathbf{f})}{v} \chi_{F}\right\|_{L^{\varepsilon}(w)} w(F)^{\frac{1}{p}-\frac{1}{\varepsilon}},
$$


where the supremum is taken over all measurable sets $F$ with $0<w(F)<\infty$. For one of such sets $F$, and $W:=w v^{-\varepsilon}$, we have that

$$
\begin{aligned}
& \left\|\frac{\mathcal{A}_{\mathcal{S}}(\mathbf{f})}{v} \chi_{F}\right\|_{L^{\varepsilon}(w)}^{\varepsilon} \leq \int_{\mathbb{R}^{n}} \sum_{Q \in \mathcal{S}} \chi_{Q}\left(\frac{\prod_{i=1}^{m} f_{Q}\left|f_{i}\right|}{v}\right)^{\varepsilon} \chi_{F} w \\
& =\sum_{Q \in \mathcal{S}}\left(\prod_{i=1}^{m} f_{Q}\left|f_{i}\right|\right)^{\varepsilon}\left(\frac{1}{W(3 Q)} \int_{Q} \chi_{F} W\right) W(3 Q)=: I .
\end{aligned}
$$

Since $W \in A_{\infty}$, there exists $r \geq 1$ such that $W \in A_{r}^{\mathcal{R}}$. Hence,

$$
\sup _{Q} \sup _{E \subseteq Q} \frac{|E|}{|Q|}\left(\frac{W(Q)}{W(E)}\right)^{1 / r}=\|W\|_{A_{r}^{\mathcal{R}}}<\infty .
$$

By hypothesis, $\mathcal{S}$ is $\eta$-sparse, so for each $Q \in \mathcal{S}$,

$$
W(3 Q) \leq\left(\frac{3^{n}}{\eta}\|W\|_{A_{r}^{\mathcal{R}}}\right)^{r} W\left(E_{Q}\right) .
$$

Using this, we get that

$$
\begin{aligned}
I & \leq\left(\frac{3^{n}}{\eta}\|W\|_{A_{r}^{\mathcal{R}}}\right)^{r} \sum_{Q \in \mathcal{S}}\left(\prod_{i=1}^{m} f_{Q}\left|f_{i}\right|\right)^{\varepsilon}\left(\frac{1}{W(3 Q)} \int_{Q} \chi_{F} W\right) W\left(E_{Q}\right) \\
& =\left(\frac{3^{n}}{\eta}\|W\|_{A_{r}^{\mathcal{R}}}\right)^{r} \sum_{Q \in \mathcal{S}} \int_{E_{Q}}\left(\prod_{i=1}^{m} f_{Q}\left|f_{i}\right|\right)^{\varepsilon}\left(\frac{1}{W(3 Q)} \int_{Q} \chi_{F} W\right) W=: I I .
\end{aligned}
$$

The sides of an $n$-dimensional cube have Lebesgue measure 0 in $\mathbb{R}^{n}$, so we can assume that the cubes in $\mathcal{S}$ are open. For $Q \in \mathcal{S}$ and $z \in E_{Q}$, we define $Q^{z}:=Q\left(z, l_{Q}\right)$, the open cube of center $z$ and side length twice the side length of $Q$. We have that $E_{Q} \subseteq Q \subseteq Q^{z} \subseteq 3 Q$, so

$$
\left(\prod_{i=1}^{m} f_{Q}\left|f_{i}\right|\right) \chi_{E_{Q}}(z) \leq \mathcal{M}(\mathbf{f})(z)
$$

and

$$
\frac{1}{W(3 Q)} \int_{Q} \chi_{F} W \leq \frac{1}{W\left(Q^{z}\right)} \int_{Q^{z}} \chi_{F} W \leq M_{W}^{c}\left(\chi_{F}\right)(z) .
$$


Since the sets $\left\{E_{Q}\right\}_{Q \in \mathcal{S}}$ are pairwise disjoint, and using Hölder's inequality with exponent $\frac{p}{\varepsilon}>1$,

$$
\begin{aligned}
I I & \leq\left(\frac{3^{n}}{\eta}\|W\|_{A_{r}^{\mathcal{R}}}\right)^{r} \int_{\mathbb{R}^{n}} \mathcal{M}(\mathbf{f})^{\varepsilon} M_{W}^{c}\left(\chi_{F}\right) W \\
& \leq\left(\frac{3^{n}}{\eta}\|W\|_{A_{r}^{\mathcal{R}}}\right)^{r}\left\|\left(\frac{\mathcal{M}(\mathbf{f})}{v}\right)^{\varepsilon}\right\|_{L^{p / \varepsilon, \infty}(w)}\left\|M_{W}^{c}\left(\chi_{F}\right)\right\|_{L^{(p / \varepsilon)^{\prime}, 1}(w)} \\
& \leq \frac{p}{p-\varepsilon}\left(\frac{3^{n}}{\eta}\|W\|_{A_{r}^{\mathcal{R}}}\right)^{r}\left\|M_{W}^{c}\right\|_{L^{(p / \varepsilon)^{\prime}, 1}(w)} w(F)^{1-\frac{\varepsilon}{p}}\left\|\frac{\mathcal{M}(\mathbf{f})}{v}\right\|_{L^{p, \infty}(w)}^{\varepsilon} .
\end{aligned}
$$

Observe that for every measurable function $g,\left\|M_{W}^{c}(g)\right\|_{L^{\infty}(w)} \leq\|g\|_{L^{\infty}(w)}$, and by standard arguments (see [27, Theorem 7.1.9]), it is easy to show that

$$
\left\|M_{W}^{c}(g)\right\|_{L^{1, \infty}(w)} \lesssim n\left[v^{-\varepsilon}\right]_{R H_{\infty}(w)}\|g\|_{L^{1}(w)} .
$$

In particular, and applying Marcinkiewicz's interpolation theorem (see [4, Theorem 4.13]), we conclude that

$$
\left\|M_{W}^{c}\right\|_{L^{(p / \varepsilon)^{\prime}, 1}(w)} \leq c_{n, p, \varepsilon}\left[v^{-\varepsilon}\right]_{R H_{\infty}(w)}^{1-\frac{\varepsilon}{p}}<\infty
$$

Combining the previous estimates, we obtain that

$$
\begin{aligned}
& \left\|\frac{\mathcal{A}_{\mathcal{S}}(\mathbf{f})}{v} \chi_{F}\right\|_{L^{\varepsilon}(w)} w(F)^{\frac{1}{p}-\frac{1}{\varepsilon}} \\
& \leq\left(\frac{p}{p-\varepsilon}\left(\frac{3^{n}}{\eta}\|W\|_{A_{r}^{\mathcal{R}}}\right)^{r} c_{n, p, \varepsilon}\left[v^{-\varepsilon}\right]_{R H_{\infty}(w)}^{1-\frac{\varepsilon}{p}}\right)^{1 / \varepsilon}\left\|\frac{\mathcal{M}(\mathbf{f})}{v}\right\|_{L^{p, \infty}(w)}
\end{aligned}
$$

and the desired result follows, with

$$
C=\inf _{r \geq 1: W \in A_{r}^{\mathcal{R}}}\left(\frac{p}{p-\varepsilon}\left(\frac{3^{n}}{\eta}\|W\|_{A_{r}^{\mathcal{R}}}\right)^{r} c_{n, p, \varepsilon}\left[v^{-\varepsilon}\right]_{R H_{\infty}(w)}^{1-\frac{\varepsilon}{p}}\right)^{1 / \varepsilon}
$$

Remark 7 For $0<p \leq 1$, if we take $v$ such that $v^{\delta} \in A_{\infty}$ for some $\delta>0$, and $w=u v^{p}$, with $u \in A_{1}$, then the previous result can be established via an extrapolation argument (see [49, Theorem 1.1]).

Under the conditions that $0<p \leq 1$, and $w=u v^{p}$, we can find weights $u$ and $v$ that satisfy the hypotheses of Theorem 1.1 in [49] but not the ones of Theorem 5 , and vice versa. If we take a non-constant weight $u \in A_{1}$, and $v=u^{-1 / p}$, then $v \in R H_{\infty} \subseteq A_{\infty}$, and $u v^{p}=1$, but for every $0<\varepsilon \leq 1$ such that $\varepsilon<p$, we have that $v^{-\varepsilon}=u^{\varepsilon / p} \in A_{1}$, and since $u$ is non-constant, $v^{-\varepsilon} \notin R H_{\infty}$. Similarly, if we take a non-constant weight $v \in A_{1}$, and $u=v^{-p}$, then $u v^{p}=1$, and for every $\varepsilon>0$, 
$u v^{p-\varepsilon}=v^{-\varepsilon} \in R H_{\infty} \subseteq A_{\infty}$, but $u \in R H_{\infty}$ and is non-constant, so $u \notin A_{1}$ (see Lemma 1).

The previous examples show that, sometimes, some of the hypotheses of Theorem 5 may be redundant. Let us be more precise on this fact. If $w \in A_{\infty}$, and $w v^{-\varepsilon}$ is a weight, then $\left[v^{-\varepsilon}\right]_{R H_{\infty}(w)}<\infty$ implies that $w v^{-\varepsilon} \in A_{\infty}$. Indeed, given a cube $Q \subseteq \mathbb{R}^{n}$, and a measurable set $E \subseteq Q$, we have that

$$
\begin{aligned}
\frac{w v^{-\varepsilon}(E)}{w v^{-\varepsilon}(Q)} & =\frac{1}{w v^{-\varepsilon}(Q)} \int_{Q} \chi_{E} w v^{-\varepsilon} \\
& \leq \frac{w(E)}{w v^{-\varepsilon}(Q)}\left\|\chi_{Q} v^{-\varepsilon}\right\|_{L^{\infty}(w)} \leq\left[v^{-\varepsilon}\right]_{R H_{\infty}(w)} \frac{w(E)}{w(Q)}
\end{aligned}
$$

and since $w \in A_{\infty}$, there exist $\delta, C>0$ such that

$$
\frac{w(E)}{w(Q)} \leq C\left(\frac{|E|}{|Q|}\right)^{\delta}
$$

so

$$
\frac{w v^{-\varepsilon}(E)}{w v^{-\varepsilon}(Q)} \leq C\left[v^{-\varepsilon}\right]_{R H_{\infty}(w)}\left(\frac{|E|}{|Q|}\right)^{\delta},
$$

and hence, $w v^{-\varepsilon} \in A_{\infty}$ (see [23]).

The next application of Theorem 2 follows from the combination of Theorems 4 and 5 , and gives us mixed restricted weak type bounds for multi-variable sparse operators that can also be deduced for other operators, such as multi-linear Calderón-Zygmund operators, using sparse domination techniques (see [37]).

Theorem 6 Let $1 \leq p_{1}, \ldots, p_{m}<\infty$, and let $\frac{1}{p}=\frac{1}{p_{1}}+\cdots+\frac{1}{p_{m}}$. Also, let $w_{1}, \ldots, w_{m}$ be weights, with $w_{i} \in A_{p_{i}}^{\mathcal{R}}, i=1, \ldots, m$, and write $v_{\mathbf{w}}=w_{1}^{p / p_{1}} \ldots w_{m}^{p / p_{m}}$. Let $v$ be a weight such that $v_{\mathbf{w}} v^{p}$ is a weight, and $w_{i} v^{p_{i}} \in A_{\infty}, i=1, \ldots, m$. Moreover, suppose that there exists $0<\varepsilon \leq 1$ such that $\varepsilon<p$, $v_{\mathbf{w}} v^{p-\varepsilon} \in A_{\infty}$, and $\left[v^{-\varepsilon}\right]_{R H_{\infty}\left(v_{\mathbf{w}} v^{p}\right)}<\infty$. Then, there exists a constant $C>0$ such that the inequality

$$
\left\|\frac{T(\mathbf{f})}{v}\right\|_{L^{p, \infty}\left(v_{\mathbf{w}} v^{p}\right)} \leq C \prod_{i=1}^{m}\left\|f_{i}\right\|_{L^{p_{i}, 1}\left(w_{i}\right)}
$$

holds for every vector of measurable functions $\mathbf{f}=\left(f_{1}, \ldots, f_{m}\right)$, where $T$ is either a sparse operator of the form

$$
\mathcal{A}_{\mathcal{S}}(\mathbf{f}):=\sum_{Q \in \mathcal{S}}\left(\prod_{i=1}^{m} f_{Q} f_{i}\right) \chi_{Q},
$$


where $\mathcal{S}$ is an $\eta$-sparse collection of dyadic cubes, or any operator that can be conveniently dominated by such sparse operators, like m-linear $\omega$-Calderón-Zygmund operators with $\omega$ satisfying the Dini condition.

Remark 8 In the case when $p_{1}=\cdots=p_{m}=1$, and $T$ is a multi-linear CalderónZygmund operator, the previous result follows from Theorem 1.9 in [40].

In general, there are examples of weights that satisfy the hypotheses of Theorem 6 apart from the constant weights. For instance, if $1 \leq p_{1}, \ldots, p_{m} \leq m^{\prime}$, we can take $w_{i}=\left(M h_{i}\right)^{\left(1-p_{i}\right) / m}$, with $h_{i} \in L_{l o c}^{1}\left(\mathbb{R}^{n}\right), i=1, \ldots, m$, and $v=v_{\mathbf{w}}^{-1 / p}$. Indeed, in virtue of Theorem 2.7 in [13], we have that $w_{i} \in A_{p_{i}}^{\mathcal{R}}, i=1, \ldots, m$, and $w_{i} v^{p_{i}}=\left(\prod_{j \neq i}\left(M h_{j}\right)^{1 / p_{j}^{\prime}}\right)^{p_{i} / m} \in A_{1}$. Observe that $v_{\mathbf{w}} v^{p}=1$, and $v=$ $\left(\prod_{i=1}^{m}\left(M h_{i}\right)^{1 / p_{i}^{\prime}}\right)^{1 / m} \in A_{1}$, so for every $\varepsilon>0, v_{\mathbf{w}} v^{p-\varepsilon}=v^{-\varepsilon} \in R H_{\infty} \subseteq A_{\infty}$.

The last application that we provide of Theorem 2 can be interpreted as a dual version of it, and generalizes [15, Proposition 2.10].

Theorem 7 Fix $p>1$, and let $u$ and $v$ be weights such that $u \in A_{p}^{\mathcal{R}}, u v^{p} \in A_{\infty}$, and for some $0<\varepsilon \leq 1, u v^{p-\varepsilon}$ is a weight and $\left[v^{-\varepsilon}\right]_{R H_{\infty}\left(u v^{p}\right)}<\infty$. Then, there exists a constant $C>0$ such that for every measurable function $f$,

$$
\left\|\frac{M\left(f u v^{p-1}\right)}{u}\right\|_{L^{p^{\prime}, \infty}(u)} \leq C\|f\|_{L^{p^{\prime}, 1}\left(u v^{p}\right)} .
$$

Proof It is known (see [32]) that there exist a collection $\left\{\mathscr{D}_{\alpha}\right\}_{\alpha}$ of $2^{n}$ general dyadic grids in $\mathbb{R}^{n}$, and a collection $\left\{\mathcal{S}_{\alpha}\right\}_{\alpha}$ of $\frac{1}{2}$-sparse families of cubes, with $\mathcal{S}_{\alpha} \subseteq \mathscr{D}_{\alpha}$, such that for every measurable function $F$,

$$
M F \leq 2 \cdot 12^{n} \sum_{\alpha=1}^{2^{n}} \mathcal{A}_{\mathcal{S}_{\alpha}}(|F|)
$$

Hence,

$$
\left\|\frac{M\left(f u v^{p-1}\right)}{u}\right\|_{L^{p^{\prime}, \infty}(u)} \leq 2 \cdot 24^{n} \sum_{\alpha=1}^{2^{n}}\left\|\frac{\mathcal{A}_{\mathcal{S}_{\alpha}}\left(|f| u v^{p-1}\right)}{u}\right\|_{L^{p^{\prime}, \infty}(u)} .
$$

By duality, and self-adjointness of $\mathcal{A}_{\mathcal{S}_{\alpha}}$, and in virtue of Hölder's inequality, we have that

$$
\begin{aligned}
\left\|\frac{\mathcal{A}_{\mathcal{S}_{\alpha}}\left(|f| u v^{p-1}\right)}{u}\right\|_{L^{p^{\prime}, \infty}(u)} & \leq p \sup _{\|g\|_{L^{p, 1}(u)} \leq 1} \int_{\mathbb{R}^{n}} \mathcal{A}_{\mathcal{S}_{\alpha}}\left(|f| u v^{p-1}\right)|g| \\
& =p \sup _{\|g\|_{L^{p, 1}(u)} \leq 1} \int_{\mathbb{R}^{n}}|f| u v^{p-1} \mathcal{A}_{\mathcal{S}_{\alpha}}(|g|)
\end{aligned}
$$




$$
\leq p \sup _{\|g\|_{L^{p, 1}(u)} \leq 1}\left\|\frac{\mathcal{A}_{\mathcal{S}_{\alpha}}(|g|)}{v}\right\|_{L^{p, \infty}\left(u v^{p}\right)}\|f\|_{L^{p^{\prime}, 1}\left(u v^{p}\right)},
$$

and the desired result follows from Theorem 5 and Theorem 2.

Remark 9 It is clear from the previous proof that Theorem 7 is also true for operators that can be conveniently dominated by sparse operators $A_{\mathcal{S}_{\alpha}}$. Even more, for a selfadjoint operator $T$, and by duality, an inequality like (4.2) follows immediately from an inequality like (1.6), with $T$ in place of $M$.

Note that for $p>1$, if $u \in A_{p}$, and $v$ is a weight, then for every measurable function $f$,

$$
\begin{aligned}
\left\|\frac{M\left(f u v^{p-1}\right)}{u}\right\|_{L^{p^{\prime}, \infty}(u)} & \leq\left\|\frac{M\left(f u v^{p-1}\right)}{u}\right\|_{L^{p^{\prime}}(u)}=\left\|M\left(f u v^{p-1}\right)\right\|_{L^{p^{\prime}\left(u^{1-p^{\prime}}\right)}} \\
& \lesssim[u]_{A_{p}}\|f\|_{L^{p^{\prime}}\left(u v^{p}\right)} \lesssim[u]_{A_{p}}\|f\|_{L^{p^{\prime}, 1}\left(u v^{p}\right)} .
\end{aligned}
$$

Hence, we obtain the conclusion of Theorem 7 without assuming that for some $0<$ $\varepsilon \leq 1,\left[v^{-\varepsilon}\right]_{R H_{\infty}\left(u v^{p}\right)}<\infty$. We would like to prove Theorem 7 without this technical hypothesis, but unfortunately, at the time of writing, we don't know how to do it.

Observe that if $v=1$, then in Theorem 7 we can take $\varepsilon=1$, and $C=C_{n, p}[u]_{A_{p}^{\mathcal{R}}}^{p+1}$, and the dependence on $u$ of the constant $C$ is explicit, although the exponent $p+1$ may not be sharp. Also, by testing on characteristic functions and using Kolmogorov's inequalities, we see that the condition that $u \in A_{p}^{\mathcal{R}}$ is necessary. This argument yields a new characterization of $A_{p}^{\mathcal{R}}$ weights and refines [15, Proposition 2.10].

Theorem 8 Fix $p>1$, and let $u$ be a weight. If $u \in A_{p}^{\mathcal{R}}$, then for every measurable function $f$,

$$
\left\|\frac{M(f u)}{u}\right\|_{L^{p^{\prime}, \infty}(u)} \lesssim n, p[u]_{A_{p}^{\mathcal{R}}}^{p+1}\|f\|_{L^{p^{\prime}, 1}(u)} .
$$

Moreover, if such an inequality holds for some constant $C>0$, then $u \in A_{p}^{\mathcal{R}}$, and $[u]_{A_{p}^{\mathcal{R}}} \leq p^{\prime} C$.

\section{Sawyer-type inequalities and multi-variable conditions on weights}

In [40, Theorem 1.5], Li, Ombrosi, and Picardi obtained an endpoint Sawyer-type inequality for the operator $\mathcal{M}$ involving $A_{\mathbf{1}}$ weights. It is natural to ask if something similar can be done in the general restricted weak setting, establishing a result for $\mathcal{M}$ like the one in Theorem 4, but assuming a multi-variable condition on the tuple of weights involved instead of imposing an individual condition on each weight. In this section we study this question. 
In view of Theorem 4 for $v=1$, it is reasonable to begin with the characterization of the weights for which the operator $\mathcal{M}$ and its centered version $\mathcal{M}^{c}$ are bounded in the restricted weak setting. This will give us the appropriate multi-variable condition on the weights. We use ideas from [16, Section 3] and [27, Theorem 7.1.9].

Theorem 9 Let $1 \leq p_{1}, \ldots, p_{m}<\infty$, and $\frac{1}{p}=\frac{1}{p_{1}}+\cdots+\frac{1}{p_{m}}$. Let $w_{1}, \ldots, w_{m}$, and $v$ be weights. The inequality

$$
\|\mathcal{M}(\mathbf{f})\|_{L^{p, \infty}(v)} \leq C \prod_{i=1}^{m}\left\|f_{i}\right\|_{L^{p_{i}, 1}\left(w_{i}\right)}
$$

holds for every vector of measurable functions $\mathbf{f}$ if, and only if

$$
[\mathbf{w}, v]_{A_{\mathbf{P}}^{\mathcal{R}}}:=\sup _{Q} v(Q)^{1 / p} \prod_{i=1}^{m} \frac{\left\|\chi \chi^{w_{i}^{-1}}\right\|_{L^{p_{i}^{\prime}, \infty}\left(w_{i}\right)}}{|Q|}<\infty .
$$

Proof First, recall that by [27, Theorem 1.4.16.(v)] (see also [3, Theorem 4.4]), we have that

$$
\frac{1}{p_{i}}\|g\|_{L^{p_{i}^{\prime}, \infty}\left(w_{i}\right)} \leq \sup \left\{\int_{\mathbb{R}^{n}}|f g| w_{i}:\|f\|_{L^{p_{i}, 1}\left(w_{i}\right)} \leq 1\right\} \leq\|g\|_{L^{p_{i}^{\prime}, \infty}\left(w_{i}\right)} .
$$

Now, fix a cube $Q$, and $\gamma>1$, and for $i=1, \ldots, m$, choose a non-negative function $f_{i}$ such that $\left\|f_{i}\right\|_{L^{p_{i}, 1}\left(w_{i}\right)} \leq 1$ and

$$
\int_{Q} f_{i}=\int_{\mathbb{R}^{n}} f_{i}\left(\chi \chi w_{i}^{-1}\right) w_{i} \geq \frac{1}{\gamma p_{i}}\left\|\chi Q w_{i}^{-1}\right\|_{L_{i}^{p_{i}^{\prime}, \infty}\left(w_{i}\right)} .
$$

Since

$$
\left(\prod_{i=1}^{m} \frac{1}{|Q|} \int_{Q}\left|f_{i}\right|\right) \chi_{Q} \leq \mathcal{M}(\mathbf{f})
$$

the hypothesis (5.1) and (5.3) imply that

$$
v(Q)^{1 / p} \prod_{i=1}^{m} \frac{\left\|\chi_{Q} w_{i}^{-1}\right\|_{L^{p_{i}^{\prime}, \infty}\left(w_{i}\right)}}{|Q|} \leq \gamma^{m} p_{1} \ldots p_{m} C,
$$

and hence, $[\mathbf{w}, v]_{A_{\mathbf{P}}^{\mathcal{R}}} \leq p_{1} \ldots p_{m} C<\infty$.

For the converse, suppose that the quantity $[\mathbf{w}, v]_{A_{\mathbf{P}}^{\mathcal{R}}}<\infty$. Observe that

$$
\mathcal{M}^{c}(\mathbf{f}) \leq \mathcal{M}(\mathbf{f}) \leq 2^{n m} \mathcal{M}^{c}(\mathbf{f}),
$$

so it suffices to establish the result for the operator $\mathcal{M}^{c}$. 
If for some $i=1, \ldots, m,\left\|f_{i}\right\|_{L^{p_{i}, 1}\left(w_{i}\right)}=\infty$, then there is nothing to prove, so we may assume that $\left\|f_{i}\right\|_{L^{p_{i}, 1}\left(w_{i}\right)}<\infty$ for every $i=1, \ldots, m$. Fix $\lambda>0$, and let $E_{\lambda}:=\left\{x \in \mathbb{R}^{n}: \mathcal{M}^{c}(\mathbf{f})(x)>\lambda\right\}$. We first show that this set is open. If for some $i=1, \ldots, m, f_{i} \notin L_{\text {loc }}^{1}\left(\mathbb{R}^{n}\right)$, then $E_{\lambda}=\mathbb{R}^{n}$. Otherwise, observe that for any $r>0$, and $x \in \mathbb{R}^{n}$, the function

$$
x \longmapsto \prod_{i=1}^{m} \frac{1}{|Q(x, r)|} \int_{Q(x, r)}\left|f_{i}\right|
$$

is continuous. Indeed, if $x_{n} \rightarrow x_{0}$, then $\left|Q\left(x_{n}, r\right)\right| \rightarrow\left|Q\left(x_{0}, r\right)\right|$, and also $\int_{Q\left(x_{n}, r\right)}\left|f_{i}\right| \rightarrow \int_{Q\left(x_{0}, r\right)}\left|f_{i}\right|$ by Lebesgue's dominated convergence theorem. Since $\left|\mathscr{Q}\left(x_{0}, r\right)\right| \neq 0$, the result follows. This implies that $\mathcal{M}^{c}(\mathbf{f})$ is the supremum of continuous functions and hence, it is lower semi-continuous, and the set $E_{\lambda}$ is open.

Given a compact subset $K$ of $E_{\lambda}$, for any $x \in K$, select an open cube $Q_{x}$ centered at $x$ such that

$$
\prod_{i=1}^{m} \frac{1}{\left|Q_{x}\right|} \int_{Q_{x}}\left|f_{i}\right|>\lambda
$$

In virtue of [27, Lemma 7.1.10], we find a subset $\left\{Q_{j}\right\}_{j=1}^{N}$ of $\left\{Q_{x}: x \in K\right\}$ such that $K \subseteq \bigcup_{j=1}^{N} Q_{j}$, and $\sum_{j=1}^{N} \chi_{Q_{j}} \leq 72^{n}$. Then, by Hölder's inequality for Lorentz spaces, (5.2), discrete Hölder's inequality with exponents $\frac{p_{i}}{p}$, and [16, Lemma 2.5],

$$
\begin{aligned}
v(K) & \leq \sum_{j=1}^{N} v\left(Q_{j}\right) \leq \frac{1}{\lambda^{p}} \sum_{j=1}^{N} v\left(Q_{j}\right)\left(\prod_{i=1}^{m} \frac{1}{\left|Q_{j}\right|} \int_{Q_{j}}\left|f_{i}\right|\right)^{p} \\
& \leq \frac{1}{\lambda^{p}} \sum_{j=1}^{N} v\left(Q_{j}\right) \prod_{i=1}^{m}\left|Q_{j}\right|^{-p}\left\|f_{i} \chi_{Q_{j}}\right\|_{L^{p_{i}, 1}\left(w_{i}\right)}^{p}\left\|\chi_{Q_{j}} w_{i}^{-1}\right\|_{L^{p_{i}^{\prime}, \infty}\left(w_{i}\right)}^{p} \\
& \left.\leq \frac{[\mathbf{w}, v]_{A_{\mathbf{P}}^{\mathcal{R}}}^{p}}{\lambda^{p}} \sum_{j=1}^{N} \prod_{i=1}^{m}\left\|f_{i} \chi_{Q_{j}}\right\|_{L^{p_{i}, 1}\left(w_{i}\right)}^{p}\right)^{p / p_{i}} \\
& \leq \frac{[\mathbf{w}, v]_{A_{\mathbf{P}}^{\mathcal{R}}}^{p}}{\lambda^{p}} \prod_{i=1}^{m}\left(\sum_{j=1}^{N}\left\|f_{i} \chi_{Q_{j}}\right\|_{L^{p_{i}, 1}\left(w_{i}\right)}^{p_{i}}\right)^{[\mathbf{w}, v]_{A_{\mathbf{P}}^{\mathcal{R}}}^{p}} \\
\prod^{p} & \leq 72^{n} \frac{f_{i} \|_{L^{p_{i}, 1}\left(w_{i}\right)}^{p}}{\lambda_{i=1}}
\end{aligned}
$$

Taking the supremum over all compact subsets $K$ of $E_{\lambda}$ and using the inner regularity of $v(x) d x$, we obtain (5.1) with constant

$$
C=2^{n m} 72^{n / p}[\mathbf{w}, v]_{A_{\mathbf{P}}^{\mathcal{R}}}
$$


Remark 10 In fact, we have proved that

$$
\frac{2^{-n m}}{\prod_{i=1}^{m} p_{i}}[\mathbf{w}, v]_{A_{\mathbf{P}}^{\mathcal{R}}} \leq\left\|\mathcal{M}^{c}\right\|_{\prod_{i=1}^{m} L^{p_{i}, 1}\left(w_{i}\right) \rightarrow L^{p, \infty}(v)} \leq 72^{n / p}[\mathbf{w}, \nu]_{A_{\mathbf{P}}^{\mathcal{R}}}
$$

and

$$
\frac{1}{\prod_{i=1}^{m} p_{i}}[\mathbf{w}, v]_{A_{\mathbf{P}}^{\mathcal{R}}} \leq\|\mathcal{M}\|_{\prod_{i=1}^{m} L^{p_{i}, 1}\left(w_{i}\right) \rightarrow L^{p, \infty}(v)} \leq 2^{n m} 72^{n / p}[\mathbf{w}, v]_{A_{\mathbf{P}}^{\mathcal{R}}}
$$

Remark 11 Observe that if $\mathcal{M}$ is bounded as in (5.1), then for every cube $Q$, if we choose $f_{1}=\cdots=f_{m}=\chi_{Q}$, we get that

$$
\left(f_{Q} v\right)^{1 / p} \leq p_{1} \ldots p_{m} C \prod_{i=1}^{m}\left(f_{Q} w_{i}\right)^{1 / p_{i}}
$$

and Lebesgue's differentiation theorem implies that $\nu \lesssim \prod_{i=1}^{m} w_{i}^{p / p_{i}}$.

In virtue of Theorem 9, we define the following class of weights.

Definition 3 Let $1 \leq p_{1}, \ldots, p_{m}<\infty$, and $\frac{1}{p}=\frac{1}{p_{1}}+\cdots+\frac{1}{p_{m}}$. Let $w_{1}, \ldots, w_{m}$, and $v$ be weights. We say that $\left(w_{1}, \ldots, w_{m}, v\right)$ belongs to the class $A_{\mathbf{P}}^{\mathcal{R}}$ if $[\mathbf{w}, v]_{A_{\mathbf{P}}^{\mathcal{R}}}<\infty$.

The condition that defines the class of $A_{\mathbf{P}}^{\mathcal{R}}$ weights depends on their behavior on cubes, and has been obtained following the ideas of Chung, Hunt, and Kurtz (see [16]). One can ask if it is possible to obtain a different condition, resembling the one obtained by Kerman and Torchinsky (see [31]). Our next theorem gives a positive answer to this question, recovering their results in the case when $m=1$ and $w_{1}=v$.

Theorem 10 Let $1 \leq p_{1}, \ldots, p_{m}<\infty$, and $\frac{1}{p}=\frac{1}{p_{1}}+\cdots+\frac{1}{p_{m}}$. Let $w_{1}, \ldots, w_{m}$, and $v$ be weights. The following statements are equivalent:

(a) $\|\mathcal{M}(\mathbf{f})\|_{L^{p, \infty}(v)} \leq C \prod_{i=1}^{m}\left\|f_{i}\right\|_{L^{p_{i}, 1}\left(w_{i}\right)}$, for every $\mathbf{f}$.

(b) $\|\mathcal{M}(\boldsymbol{\varnothing})\|_{L^{p, \infty}(v)} \leq c \prod_{i=1}^{m} w_{i}\left(E_{i}\right)^{1 / p_{i}}$, for every $\boldsymbol{\emptyset}=\left(\chi_{E_{1}}, \ldots, \chi_{E_{m}}\right)$.

(c)

$$
\|\mathbf{w}, v\|_{A_{\mathbf{P}}^{\mathcal{R}}}:=\sup _{Q} v(Q)^{1 / p} \prod_{i=1}^{m} \sup _{0<w_{i}\left(E_{i}\right)<\infty} \frac{\left|E_{i} \cap Q\right|}{|Q|} w_{i}\left(E_{i}\right)^{-1 / p_{i}}<\infty .
$$

(d) $\left(w_{1}, \ldots, w_{m}, v\right) \in A_{\mathbf{P}}^{\mathcal{R}}$.

Moreover, if $\left(w_{1}, \ldots, w_{m}, v\right) \in A_{\mathbf{P}}^{\mathcal{R}}$, and $v \in A_{\infty}$, then

$$
T: L^{p_{1}, 1}\left(w_{1}\right) \times \cdots \times L^{p_{m}, 1}\left(w_{m}\right) \longrightarrow L^{p, \infty}(v),
$$


where $T$ is either a sparse operator as in (4.1), or any operator that can be conveniently dominated by such sparse operators, like m-linear $\omega$-Calderón-Zygmund operators with $\omega$ satisfying the Dini condition.

Proof It is clear that (a) implies (b), and we have already proved in Theorem 9 that (a) and (d) are equivalent. Let us show that (b) implies (c). Fix a cube $Q$ and measurable sets $E_{i}$, for $i=1, \ldots, m$, with $0<w_{i}\left(E_{i}\right)<\infty$. Since

$$
\left(\prod_{i=1}^{m} \frac{\left|E_{i} \cap Q\right|}{|Q|}\right) \chi_{Q} \leq \mathcal{M}(\boldsymbol{\varnothing})
$$

we apply (b) to conclude that

$$
v(Q)^{1 / p} \prod_{i=1}^{m} \frac{\left|E_{i} \cap Q\right|}{|Q|} \leq c \prod_{i=1}^{m} w_{i}\left(E_{i}\right)^{1 / p_{i}}
$$

and hence, $\|\mathbf{w}, v\|_{A_{\mathbf{P}}^{\mathcal{R}}} \leq c<\infty$.

We now prove that (c) is equivalent to (d). First, observe that for every $i=1, \ldots, m$,

$$
\sup _{0<w_{i}\left(E_{i}\right)<\infty} \frac{\left|E_{i} \cap Q\right|}{w_{i}\left(E_{i}\right)^{1 / p_{i}}}=\sup _{E_{i} \subseteq Q} \frac{\left|E_{i}\right|}{w_{i}\left(E_{i}\right)^{1 / p_{i}}},
$$

where the first supremum is taken over all measurable sets $E_{i}$ such that $0<w_{i}\left(E_{i}\right)<$ $\infty$, and the second one is taken over all non-empty measurable sets $E_{i} \subseteq Q$. Now, in virtue of [16, Lemma 2.8] and Kolmogorov's inequalities, we have that

$$
\left\|\chi_{Q} w_{i}^{-1}\right\|_{L^{p_{i}^{\prime}, \infty}{ }_{\left(w_{i}\right)}} \leq \sup _{E_{i} \subseteq Q} \frac{\left|E_{i}\right|}{w_{i}\left(E_{i}\right)^{1 / p_{i}}} \leq p_{i}\left\|\chi \chi_{Q} w_{i}^{-1}\right\|_{L^{p_{i}^{\prime}, \infty}\left(w_{i}\right)},
$$

and hence, $[\mathbf{w}, v]_{A_{\mathbf{P}}^{\mathcal{R}}} \leq\|\mathbf{w}, v\|_{A_{\mathbf{P}}^{\mathcal{R}}} \leq p_{1} \ldots p_{m}[\mathbf{w}, v]_{A_{\mathbf{P}}^{\mathcal{R}}}$.

Note that a similar argument to the one in the proof of Theorem 5 shows that for $0<\varepsilon \leq 1$ such that $\varepsilon<p$, and $r \geq 1$ such that $\nu \in A_{r}^{\mathcal{R}}$,

$$
\left\|\mathcal{M}_{\mathcal{S}}(\mathbf{f})\right\|_{L^{p, \infty}(v)} \leq\left\|\mathcal{A}_{\mathcal{S}}(|\mathbf{f}|)\right\|_{L^{p, \infty}(v)} \leq C_{\varepsilon, \eta, n, p, r}[v]_{A_{r}^{\mathcal{R}}}^{r / \varepsilon}\left\|\mathcal{M}_{\mathcal{S}}(\mathbf{f})\right\|_{L^{p, \infty}(v)},
$$

where

$$
\mathcal{M}_{\mathcal{S}}(\mathbf{f}):=\sup _{Q \in \mathcal{S}}\left(\prod_{i=1}^{m} f_{Q}\left|f_{i}\right|\right) \chi_{Q}
$$

and since $\mathcal{S}$ is a countable collection of dyadic cubes, the proof of Theorem 9 can be rewritten to show that

$$
\mathcal{M}_{\mathcal{S}}: L^{p_{1}, 1}\left(w_{1}\right) \times \cdots \times L^{p_{m}, 1}\left(w_{m}\right) \longrightarrow L^{p, \infty}(v)
$$


if, and only if

$$
[\mathbf{w}, v]_{A_{\mathbf{P}, \mathcal{S}}^{\mathcal{R}}}:=\sup _{Q \in \mathcal{S}} v(Q)^{1 / p} \prod_{i=1}^{m} \frac{\left\|\chi \chi w_{i}^{-1}\right\|_{L^{p_{i}^{\prime}, \infty}\left(w_{i}\right)}}{|Q|}<\infty
$$

which is true, since $[\mathbf{w}, v]_{A_{\mathbf{P}, \mathcal{S}}^{\mathcal{R}}} \leq[\mathbf{w}, v]_{A_{\mathbf{P}}^{\mathcal{R}}}<\infty$. Moreover,

$$
\frac{1}{\prod_{i=1}^{m} p_{i}}[\mathbf{w}, v]_{A_{\mathbf{P}, \mathcal{S}}^{\mathcal{R}}} \leq\left\|\mathcal{M}_{\mathcal{S}}\right\|_{\prod_{i=1}^{m} L^{p_{i}, 1}\left(w_{i}\right) \rightarrow L^{p, \infty}(v)} \leq[\mathbf{w}, v]_{A_{\mathbf{P}, \mathcal{S}}^{\mathcal{R}}},
$$

so (5.5) implies that

$$
\frac{1}{\prod_{i=1}^{m} p_{i}}[\mathbf{w}, v]_{A_{\mathbf{P}, \mathcal{S}}^{\mathcal{R}}} \leq\left\|\mathcal{A}_{\mathcal{S}}\right\|_{\prod_{i=1}^{m} L^{p_{i}, 1}\left(w_{i}\right) \rightarrow L^{p, \infty}(\nu)} \leq C_{\varepsilon, \eta, n, p, r}[\nu]_{A_{r}^{\mathcal{R}}}^{r / \varepsilon}[\mathbf{w}, \nu]_{A_{\mathbf{P}, \mathcal{S}}^{\mathcal{R}}} .
$$

Finally, in virtue of Theorem 1.2 and Proposition 3.1 in [37] (see also [33, Theorem 3.1]), if $T$ is an $m$-linear $\omega$-Calderón-Zygmund operator with $\omega$ satisfying the Dini condition, then there exists a dimensional constant $0<\eta<1$ such that given compactly supported functions $f_{i} \in L^{1}\left(\mathbb{R}^{n}\right), i=1, \ldots, m$, there exists an $\eta$-sparse collection of dyadic cubes $\mathcal{S}$ such that

$$
\left|T\left(f_{1}, \ldots, f_{m}\right)\right| \leq c_{n} C_{T} \mathcal{A}_{\mathcal{S}}(|\mathbf{f}|)
$$

Hence, (5.4) follows from (5.6) and the standard density argument in [27, Exercise 1.4.17]. Moreover,

$$
\|T\|_{\prod_{i=1}^{m} L^{p_{i}, 1}\left(w_{i}\right) \rightarrow L^{p, \infty}(v)} \leq c_{n} C_{T} C_{\varepsilon, \eta, n, p, r}[\nu]_{A_{r}^{\mathcal{R}}}^{r / \varepsilon}[\mathbf{w}, \nu]_{A_{\mathbf{P}}^{\mathcal{R}}}
$$

Remark 12 Given weights $w_{1}, \ldots, w_{m}$, and $v=\prod_{i=1}^{m} w_{i}^{p / p_{i}}$, the equivalence between (b) and (c) in Theorem 10 can be found in [5]. Moreover, if $p_{1}=\cdots=$ $p_{m}=1$, then the equivalence between (a) and (d) can be found in [36]. Observe that if $\mathbf{w} \in A_{\mathbf{P}}$, then $\left(w_{1}, \ldots, w_{m}, v_{\mathbf{w}}\right) \in A_{\mathbf{P}}^{\mathcal{R}}$. In [36], strong and weak type bounds for $m$-linear Calderón-Zygmund operators were established for the first time for tuples of weights in $A_{\mathbf{P}}$. In [42], these results were extended to $m$-linear $\omega$-Calderón-Zygmund operators with $\|\omega\|_{\text {Dini }}<\infty$.

We can now state our main conjecture on Sawyer-type inequalities with $A_{\mathbf{P}}^{\mathcal{R}}$ weights, a complete multi-variable version of Theorem 2 for $\mathcal{M}$.

Conjecture 1 Let $1 \leq p_{1}, \ldots, p_{m}<\infty$, and let $\frac{1}{p}=\frac{1}{p_{1}}+\cdots+\frac{1}{p_{m}}$. Let $w_{1}, \ldots, w_{m}$, and $v$ be weights, and suppose that $\left(w_{1}, \ldots, w_{m}, v\right) \in A_{\mathbf{P}}^{\mathcal{R}}$. Let $v$ be a weight such that $\nu v^{p} \in A_{\infty}$. Then, there exists a constant $C>0$ such that the inequality 


$$
\left\|\frac{\mathcal{M}(\mathbf{f})}{v}\right\|_{L^{p, \infty}\left(\nu v^{p}\right)} \leq C \prod_{i=1}^{m}\left\|f_{i}\right\|_{L^{p_{i}, 1}\left(w_{i}\right)}
$$

holds for every vector of measurable functions $\mathbf{f}=\left(f_{1}, \ldots, f_{m}\right)$.

Remark 13 This conjecture is true in the case when $p_{1}=\cdots=p_{m}=1$ and $v=v_{\mathbf{w}}$, as shown in [40, Theorem 1.5]. We don't know if the hypothesis that $\nu v^{p} \in A_{\infty}$ can be replaced by $v^{\delta} \in A_{\infty}$ for some $\delta>0$.

In virtue of Hölder's inequality, if $\mathbf{w} \in \prod_{i=1}^{m} A_{p_{i}}^{\mathcal{R}}$, then $\left(w_{1}, \ldots, w_{m}, v_{\mathbf{w}}\right) \in A_{\mathbf{P}}^{\mathcal{R}}$, so this conjecture extends the result for $\mathcal{M}$ presented in Theorem 4. Also, combining such conjecture with Theorem 5, we would get a generalization of Theorem 6 in the line of [40, Theorem 1.9].

As it happens in the one-dimensional case, the conclusion of Conjecture 1 is completely elementary if $1<p_{1}, \ldots, p_{m}<\infty, \mathbf{w} \in A_{\mathbf{P}}$, and $v=v_{\mathbf{w}}$, since

$$
\begin{aligned}
& \left\|\frac{\mathcal{M}(\mathbf{f})}{v}\right\|_{L^{p, \infty}\left(v_{\mathbf{w}} v^{p}\right)} \leq\left\|\frac{\mathcal{M}(\mathbf{f})}{v}\right\|_{L^{p}\left(v_{\mathbf{w}} v^{p}\right)}=\|\mathcal{M}(\mathbf{f})\|_{L^{p}\left(v_{\mathbf{w}}\right)} \\
& \lesssim[\mathbf{w}]_{A \mathbf{P}}^{\max \left\{\frac{p_{1}^{\prime}}{p}, \ldots, \frac{p_{m}^{\prime}}{p}\right\}} \prod_{i=1}^{m}\left\|f_{i}\right\|_{L^{p_{i}\left(w_{i}\right)}} \lesssim[\mathbf{w}]_{A_{\mathbf{P}}}^{\max \left\{\frac{p_{1}^{\prime}}{p}, \ldots, \frac{p_{m}^{\prime}}{p}\right\}} \prod_{i=1}^{m}\left\|f_{i}\right\|_{L^{p_{i}, 1}\left(w_{i}\right)},
\end{aligned}
$$

where we have used the sharp estimates for $\mathcal{M}$ proved in [38, Theorem 1.2].

In the general case, observe that for every $\theta>0$,

$$
\begin{aligned}
& \mathcal{M}(\mathbf{f}) \leq[\mathbf{w}, v]_{A_{\mathbf{P}}^{\mathcal{R}}} \sup _{Q} \frac{\chi_{Q}}{v(Q)^{1 / p}} \prod_{i=1}^{m}\left\|f_{i} \chi_{Q}\right\|_{L^{p_{i}, 1}\left(w_{i}\right)} \\
& =[\mathbf{w}, v]_{A_{\mathbf{P}}^{\mathcal{R}}}\left(\sup _{Q} \frac{\chi_{Q}}{v(Q)^{\theta / p}} \prod_{i=1}^{m}\left\|f_{i} \chi_{Q}\right\|_{L^{p_{i}, 1}\left(w_{i}\right)}^{\theta}\right)^{1 / \theta}=:[\mathbf{w}, v]_{A_{\mathbf{P}}^{\mathcal{R}}} \mathcal{N}_{\mathbf{w}, v}^{\theta}(\mathbf{f})^{1 / \theta},
\end{aligned}
$$

and

$$
\left\|\frac{\mathcal{M}(\mathbf{f})}{v}\right\|_{L^{p, \infty}\left(v v^{p}\right)} \leq[\mathbf{w}, v]_{A_{\mathbf{P}}^{\mathcal{R}}}\left\|\frac{\mathcal{N}_{\mathbf{w}, v}^{\theta}(\mathbf{f})}{V}\right\|_{L^{p / \theta, \infty}\left(v V^{p / \theta}\right)}^{1 / \theta},
$$

with $V:=v^{\theta}$. We suspect that a wise choice of $\theta$ (maybe $\theta=p$ or $\theta=m p$ ) and the argument in the proof of Theorem 1.5 in [40] could lead to some advances towards our conjecture. This idea requires further investigation.

Acknowledgements E. R. P. wants to express his sincere gratitude to David V. Cruz-Uribe for his help and efforts when we were trying to weaken the hypotheses of Theorem 2. The authors thank M. J. Carro for her suggestions to improve this document. They also thank the reviewer for the feedback, which led to the release of Sect. 5. 
Funding Funding: This work was supported by the Spanish Ministry of Economy and Competitiveness [MTM2017-82160-C2-2-P and SEV-2017-0718 to C. P.]; and the Spanish Ministry of Education, Culture and Sports [FPU14/04463 to E. R. P.].

\section{Declarations}

Conflict of interest The authors declare that they have no conflict of interest.

Open Access This article is licensed under a Creative Commons Attribution 4.0 International License, which permits use, sharing, adaptation, distribution and reproduction in any medium or format, as long as you give appropriate credit to the original author(s) and the source, provide a link to the Creative Commons licence, and indicate if changes were made. The images or other third party material in this article are included in the article's Creative Commons licence, unless indicated otherwise in a credit line to the material. If material is not included in the article's Creative Commons licence and your intended use is not permitted by statutory regulation or exceeds the permitted use, you will need to obtain permission directly from the copyright holder. To view a copy of this licence, visit http://creativecommons.org/licenses/by/4.0/.

\section{References}

1. Álvarez, J., Bagby, R.J., Kurtz, D.S., Pérez, C.: Weighted estimates for commutators of linear operators. Studia Math. 104(2), 195-209 (1993)

2. Andersen, K.F., Muckenhoupt, B.: Weighted weak type Hardy inequalities with applications to Hilbert transforms and maximal functions. Studia Math. 72(1), 9-26 (1982)

3. Barza, S., Kolyada, V., Soria, J.: Sharp constants related to the triangle inequality in Lorentz spaces. Trans. Am. Math. Soc. 361(10), 5555-5574 (2009)

4. Bennett, C., Sharpley, R.: Interpolation of operators. Pure and Applied Mathematics, 129, Academic Press, Inc., Boston, MA (1988)

5. Bernardis, A.L., Crescimbeni, R., Martín-Reyes, F.J.: Multilinear Cesàro maximal operators. J. Math. Anal. Appl. 397(1), 191-204 (2013)

6. Berra, F.: Mixed weak estimates of Sawyer type for generalized maximal operators. Proc. Am. Math. Soc. 147(10), 4259-4273 (2019)

7. Berra, F., Carena, M., Pradolini, G.: Mixed weak estimates of Sawyer type for commutators of generalized singular integrals and related operators. Michigan Math. J. 68(3), 527-564 (2019)

8. Berra, F., Carena, M., Pradolini, G.: Mixed weak estimates of Sawyer type for fractional integrals and some related operators. J. Math. Anal. Appl. 479(2), 1490-1505 (2019)

9. Berra, F., Carena, M., Pradolini, G.: Improvements on Sawyer type estimates for generalized maximal functions. Math. Nachr. 293(10), 1911-1930 (2020)

10. Buckley, S.: Estimates for operator norms on weighted spaces and reverse Jensen inequalities. Trans. Am. Math. Soc. 340(1), 253-272 (1993)

11. Caldarelli, M., Rivera-Ríos, I.P.: A sparse approach to mixed weak type inequalities. Math. Z. 296(1-2), 787-812 (2020)

12. Cao, M., Xue, Q., Yabuta, K.: Weak and strong type estimates for the multilinear pseudo-differential operators. J. Funct. Anal. 278, no. 10, 108454, 46 pp (2020)

13. Carro, M.J., Grafakos, L., Soria, J.: Weighted weak-type $(1,1)$ estimates via Rubio de Francia extrapolation. J. Funct. Anal. 269, 1203-1233 (2015)

14. Carro, M.J., Roure-Perdices, E.: Weighted boundedness of the 2-fold product of Hardy-Littlewood maximal operators. Math. Nachr. 291(8-9), 1208-1215 (2018)

15. Carro, M.J., Soria, J.: Restricted weak-type Rubio de Francia extrapolation for $p>p_{0}$ with applications to exponential integrability estimates. Adv. Math. 290, 888-918 (2016)

16. Chung, H.H., Hunt, R., Kurtz, D.S.: The Hardy-Littlewood maximal function on $L(p, q)$ spaces with weights. Indiana Univ. Math. J. 31, 109-120 (1982)

17. Coifman, R. R., Rochberg, R., Weiss, G.: Factorization theorems for Hardy spaces in several variables. Ann. Math. (2) 103, no. 3, 611-635 (1976)

18. Cruz-Uribe, D., Pérez, C.: Two weight extrapolation via the maximal operator. J. Funct. Anal. 174(1), $1-17$ (2000) 
19. Cruz-Uribe, D., Martell, J.M., Pérez, C.: Weighted weak-type inequalities and a conjecture of Sawyer. Int. Math. Res. Not., no. 30, 1849-1871 (2005)

20. Cruz-Uribe, D., Martell, J.M., Pérez, C.: Weights, extrapolation and the theory of Rubio de Francia. Operator Theory: Advances and Applications, 215, Birkhäuser/Springer Basel AG, Basel (2011)

21. Cruz-Uribe, D., Neugebauer, C.J.: The structure of the reverse Hölder classes. Trans. Am. Math. Soc. 347(8), 2941-2960 (1995)

22. Curbera, G.P., García-Cuerva, J., Martell, J.M., Pérez, C.: Extrapolation with weights, rearrangementinvariant function spaces, modular inequalities and applications to singular integrals. Adv. Math. 203(1), 256-318 (2006)

23. Duoandikoetxea, J., Martín-Reyes, F.J., Ombrosi, S.: On the $A_{\infty}$ conditions for general bases. Math. Z. 282(3-4), 955-972 (2016)

24. Edmunds, D.E., Kokilashvili, V., Meskhi, A.: Bounded and compact integral operators. Mathematics and its Applications, 543, Kluwer Academic Publishers, Dordrecht (2002)

25. Fujii, N.: Weighted bounded mean oscillation and singular integrals. Math. Jpn. 22 (1977/78), no. 5, 529-534

26. García-Cuerva, J., Rubio de Francia, J.L.: Weighted norm inequalities and related topics. North-Holland Mathematics Studies, 116, North-Holland Publishing Co., Amsterdam (1985)

27. Grafakos, L.: Classical Fourier analysis. Third edition. Graduate Texts in Mathematics, 249, Springer, New York (2014)

28. Grafakos, L., Torres, R.H.: Multilinear Calderón-Zygmund theory. Adv. Math. 165(1), 124-164 (2002)

29. Hytönen, T., Pérez, C.: Sharp weighted bounds involving $A_{\infty}$. Anal. PDE 6(4), 777-818 (2013)

30. Hytönen, T., Pérez, C., Rela, E.: Sharp reverse Hölder property for $A_{\infty}$ weights on spaces of homogeneous type. J. Funct. Anal. 263(12), 3883-3899 (2012)

31. Kerman, R.A., Torchinsky, A.: Integral inequalities with weights for the Hardy maximal function. Studia Math. 71(3), 277-284 (1982)

32. Lerner, A. K.: A simple proof of the $A_{2}$ conjecture. Int. Math. Res. Not., no. 14, 3159-3170 (2013)

33. Lerner, A.K.: On pointwise estimates involving sparse operators. New York J. Math. 22, 341-349 (2016)

34. Lerner, A.K., Nazarov, F.: Intuitive dyadic calculus: the basics. Expo. Math. 37(3), 225-265 (2019)

35. Lerner, A.K., Ombrosi, S., Pérez, C.: Weak type estimates for singular integrals related to a dual problem of Muckenhoupt-Wheeden. J. Fourier Anal. Appl. 15(3), 394-403 (2009)

36. Lerner, A.K., Ombrosi, S., Pérez, C., Torres, R.H., Trujillo-González, R.: New maximal functions and multiple weights for the multilinear Calderón-Zygmund theory. Adv. Math. 220(4), 1222-1264 (2009)

37. Li, K.: Sparse Domination Theorem for Multilinear Singular Integral Operators with $L^{r}$-Hörmander Condition. Michigan Math. J. 67(2), 253-265 (2018)

38. Li, K., Moen, K., Sun, W.: The sharp weighted bound for multilinear maximal functions and CalderónZygmund operators. J. Fourier Anal. Appl. 20(4), 751-765 (2014)

39. Li, K., Ombrosi, S., Pérez, C.: Proof of an extension of E. Sawyer's conjecture about weighted mixed weak-type estimates. Math. Ann. 374, no. 1-2, 907-929 (2019)

40. Li, K., Ombrosi, S., Picardi, B.: Weighted mixed weak-type inequalities for multilinear operators. Studia Math. 244(2), 203-215 (2019)

41. Lorente, M., Martín-Reyes, F.J.: Some mixed weak type inequalities. J. Math. Inequal. 15(2), 811-826 (2021)

42. Lu, G., Zhang, P.: Multilinear Calderón-Zygmund operators with kernels of Dini's type and applications. Nonlinear Anal. 107, 92-117 (2014)

43. Martín-Reyes, F.J., Ombrosi, S.: Mixed weak type inequalities for one-sided operators. Q. J. Math. 60(1), 63-73 (2009)

44. Martín-Reyes, F.J., Ortega Salvador, P., Sarrión Gavilán, M.D.: Boundedness of operators of Hardy type in $\Lambda^{p, q}$ spaces and weighted mixed inequalities for singular integral operators. Proc. R. Soc. Edinburgh Sect. A 127(1), 157-170 (1997)

45. Muckenhoupt, B.: Weighted norm inequalities for the Hardy maximal function. Trans. Am. Math. Soc. 165, 207-226 (1972)

46. Muckenhoupt, B.: The equivalence of two conditions for weight functions. Studia Math. 49 (1973/74), 101-106

47. Muckenhoupt, B.: Weighted norm inequalities for classical operators. Harmonic analysis in Euclidean spaces (Proc. Symp. Pure Math. Am. Math. Soc., Williamstown, Mass., 1978, Part I), pp. 69-83, Providence, R. I., (1979) 
48. Muckenhoupt, B., Wheeden, R.: Some weighted weak-type inequalities for the Hardy-Littlewood maximal function and the Hilbert transform. Indiana Univ. Math. J. 26, 801-816 (1977)

49. Ombrosi, S., Pérez, C.: Mixed weak type estimates: examples and counterexamples related to a problem of E. Sawyer. Colloq. Math. 145(2), 259-272 (2016)

50. Ombrosi, S., Pérez, C., Recchi, J.: Quantitative weighted mixed weak-type inequalities for classical operators. Indiana Univ. Math. J. 65(2), 615-640 (2016)

51. Osȩkowski, A., Rapicki, M.: A weighted maximal weak-type inequality. Mathematika 67(1), 145-157 (2021)

52. Picardi B.: Weighted mixed weak-type inequalities for multilinear fractional operators. Oct. 15, 2018. arXiv:1810.06680 [math.CA]

53. Roure-Perdices, E.: Restricted Weak Type Extrapolation of Multi-Variable Operators and Related Topics. PhD Dissertation, University of Barcelona, 2019. Available at http://hdl.handle.net/2445/148841, and http://hdl.handle.net/10803/668407

54. Sawyer, E.T.: A weighted weak type inequality for the maximal function. Proc. Am. Math. Soc. 93, 610-614 (1985)

55. Stockdale, C. B.: A different approach to endpoint weak-type estimates for Calderón-Zygmund operators. J. Math. Anal. Appl. 487 (2020), no. 2, 124016, 13 pp

56. Wen, Y., Wu, H., Zhang, J.: Weighted variation inequalities for singular integrals and commutators. $J$. Math. Anal. Appl. 485, no. 2, 123825, 16 pp (2020)

Publisher's Note Springer Nature remains neutral with regard to jurisdictional claims in published maps and institutional affiliations. 